\title{
Dynamics of meromorphic mappings with small topological degree II: Energy and invariant measure
}

\author{
Jeffrey Diller, Romain Dujardin and Vincent Guedj
}

\begin{abstract}
We continue our study of the dynamics of meromorphic mappings with small topological degree $\lambda_{2}(f)<\lambda_{1}(f)$ on a compact Kähler surface $X$. Under general hypotheses we are able to construct a canonical invariant measure which is mixing, does not charge pluripolar sets and has a natural geometric description.

Our hypotheses are always satisfied when $X$ has Kodaira dimension zero, or when the mapping is induced by a polynomial endomorphism of $\mathbb{C}^{2}$. They are new even in the birational case $\left(\lambda_{2}(f)=1\right)$. We also exhibit families of mappings where our assumptions are generically satisfied and show that if counterexamples exist, the corresponding measure must give mass to a pluripolar set.
\end{abstract}

Mathematics Subject Classification (2010). 37F10, 32H50, 32U40.

Keywords. Complex dynamics, meromorphic mappings, geometric currents, intersection of positive closed currents.

\section{Introduction}

In this article we continue from [DDG1] our study of the dynamics of meromorphic mappings with small topological degree on complex surfaces. Whereas our previous article focused on constructing canonical forward and backward invariant currents for a given mapping, here we take up the problem of intersecting these currents to create a natural invariant measure. In fact, the first half of this article does not concern dynamics at all but rather the general problem of defining the wedge product of two positive closed $(1,1)$ currents on a compact complex surface. This is of interest in its own right, and our treatment draws much in content and spirit from recent work of Guedj and Zeriahi [GZ2] concerning the definition of the complex MongeAmpère operator in the compact setting. Let us begin, nevertheless, by rehearsing the dynamical setting of immediate concern. We refer the reader to [DDG1] for a more thorough presentation and list of references.

Let $(X, \omega)$ be a compact Kähler surface and $f: X \rightarrow X$ a meromorphic selfmap. We assume that $f$ is 1 -stable, meaning that the induced action $f^{*}: H^{1,1}(X) \rightarrow$ 
$H^{1,1}(X)$ satisfies

$$
\left(f^{n}\right)^{*}=\left(f^{*}\right)^{n} \quad \text { for all } n \in \mathbb{N} .
$$

We let $\lambda_{2}=\lambda_{2}(f)$ denote the topological degree of $f$ and $\lambda_{1}=\lambda_{1}(f)$ be the first dynamical degree of $f$. These are the spectral radii of the actions $f^{*}: H^{j, j}(X) \rightarrow$ $H^{j, j}(X)$ for $j=2$ and $j=1$, respectively. Our main assumption is that $f$ has small topological degree; that is,

$$
\lambda_{2}(f)<\lambda_{1}(f) .
$$

Notice that with this terminology a birational map $\left(\lambda_{2}=1\right)$ has small topological degree only when $\lambda_{1}>1$. Note also that in this case either $X$ is rational or $\operatorname{kod}(X)=0$.

Under these conditions we have shown in [DDG1] that there exist closed positive $(1,1)$ currents $T^{+}$and $T^{-}$uniquely characterized by

$$
T^{+}=\lim _{n \rightarrow \infty} \frac{1}{\lambda_{1}^{n}}\left(f^{n}\right)^{*} \omega \quad \text { and } \quad T^{-}=\lim _{n \rightarrow \infty} \frac{1}{\lambda_{1}^{n}}\left(f^{n}\right)_{*} \omega .
$$

Furthermore, $T^{ \pm}$can be written as $T^{ \pm}=\omega^{ \pm}+d d^{c} G^{ \pm}$, where $\omega^{ \pm}$are positive closed currents with bounded potentials. By scaling $\omega$, we normalize the cohomological intersection number so that $\left\{T^{+}\right\} \cdot\left\{T^{-}\right\}=1$. Finally, under the additional assumption that $X$ is projective (non-projective examples can arise on surfaces of Kodaira dimension zero), the invariant currents have special geometric properties: $T^{+}$is laminar while $T^{-}$is woven (see [DDG1] for definitions).

We have three goals in this article.

- First, we seek general conditions under which the wedge product $T^{+} \wedge T^{-}$may be reasonably defined as a probability measure $\mu_{f}$ on $X$. Here, "reasonably defined" is understood as a continuity requirement: when $T^{ \pm}$are approximated in a standard pluripotential theoretic sense by convergent sequences $\left(T_{j}^{ \pm}\right)$of less singular positive closed $(1,1)$ currents, then we insist that $\lim _{j \rightarrow \infty} T_{j}^{+} \wedge T_{j}^{-} \rightarrow \mu_{f}$.

- Second, we seek to show that the conditions that guarantee existence of $\mu_{f}$ also ensure that it has good dynamical properties:

(i) $\mu_{f}$ is invariant under $f$;

(ii) $\mu_{f}$ is mixing;

(iii) $\mu_{f}$ may be alternately viewed as geometric intersection of the laminar/ woven structures of the currents $T^{+}$and $T^{-}$.

In a third article, [DDG3] we will greatly elaborate on this second goal, studying the fine ergodic properties of $\left(f, \mu_{f}\right)$ when properties (i) to (iii) are satisfied. 
- Finally we seek to apply our results to particular examples of meromorphic mappings. This requires that we find checkable dynamical criteria that imply the potential theoretic conditions needed to define $\mu_{f}$.

It should be noted that, as with the other two articles [DDG1], [DDG3] in this series, this one represents an attempt to generalize things that are known about birational maps to the larger setting of maps with small topological degree. On the other hand, this paper is the only one in the series that gives new results even in the birational setting.

Let us now describe our results. Regarding the first goal, let $S=\alpha+d d^{c} \varphi$ and $T=\beta+d d^{c} \psi$ be any two positive closed $(1,1)$ currents on $X$, each expressed in terms of a positive current with bounded local potentials $(\alpha, \beta)$ and a negative (relative) potential function $(\varphi, \psi)$. Products $\alpha \wedge T$ involving a positive current with bounded potentials are well-understood [BT1]. The most straightforward way to define $S \wedge T$ more generally is to require $\varphi \in L^{1}(T)$ and then declare $d d^{c} \varphi \wedge T:=d d^{c}(\varphi T)$. We write " $T \in L^{1}(S)$ " when this integrability holds. This condition is independent of the choice of decomposition of $T$ and moreover symmetric in $S$ and $T$.

In our dynamical situation, the condition $T^{+} \in L^{1}\left(T^{-}\right)$suffices for our first goal. However, it is not clear that the resulting measure $\mu_{f}$ then has good dynamical properties. In particular it seems difficult, given only the $L^{1}$ condition, to establish that the intersection $T^{+} \wedge T^{-}$is geometric. Moreover, in certain cases (see e.g. the examples in §4.4) we do not know whether $T^{+} \in L^{1}\left(T^{-}\right)$, but we are nevertheless able to define a "reasonable" wedge product $T^{+} \wedge T^{-}$by taking advantage of the global (compact Kähler) context, in the spirit of [GZ2].

Let $S$ and $T$ be positive closed currents as before. We say that $S$ has finite $T$ energy, and denote " $S \in \mathcal{E}(T)$ ", if there exists an unbounded convex increasing function $\chi:(-\infty, 0] \rightarrow(-\infty, 0]$ such that $\chi \circ \varphi \in L^{1}(\alpha \wedge T)$ and the "weighted energy"

$$
\int \chi^{\prime} \circ \varphi d \varphi \wedge d^{c} \varphi \wedge T
$$

is finite. This condition is independent of the choice of decomposition for $S$. We stress that it does not imply that $S \in L^{1}(T)$.

Our first main result is the following.

Theorem 1. Let $S=\alpha+d d^{c} \varphi$ and $T=\beta+d d^{c} \psi$ be positive closed currents on a compact Kähler surface as above. Assume that $S \in \mathcal{E}(T), T \in \mathcal{E}(S)$ and $T$ does not charge pluripolar sets.

Then the product $S \wedge T$ is well-defined and does not charge pluripolar sets.

When the first assumption of this theorem holds for the invariant currents $T^{+}$ and $T^{-}$associated to a 1-stable meromorphic map $f: X \rightarrow X$ of small topological degree, we say that $f$ has finite dynamical energy. In this case we can show that the 
current $T^{+}$does not charge pluripolar sets (see Proposition 2.2). Thus the measure $\mu_{f}=T^{+} \wedge T^{-}$is well defined and does not charge pluripolar sets. We are further able to prove that $\mu_{f}$ has the desired properties (i)-(iii) above, property (iii) being the hardest to establish.

Theorem 2. Let $f$ be a 1-stable meromorphic self map of a compact Kähler surface, with small topological degree. Assume that $f$ has finite dynamical energy. Then $\mu_{f}:=T^{+} \wedge T^{-}$is invariant and mixing.

If furthermore $X$ is projective then $\mu_{f}$ is described by the geometric intersection of the laminar/woven structures of $T^{+/-}$.

We now turn to the problem of checking the finite dynamical energy condition; i.e., of finding a weight $\chi$ so that the integral in (2) is demonstrably finite. Some weights are, of course, easier to work with than others. The class of homogeneous weights $\chi(t)=-(-t)^{p}, 0<p \leq 1$ turns out to be particularly useful for dynamical applications.

For the weight $\chi(t)=t$ we obtain the following criterion which generalizes the work of Bedford and the first author [BD] (see also [DG]). In order to state it, we let $I^{+}$denote the indeterminacy set of $f$, and $I^{-}=f\left(E^{+}\right)$denote the image of the exceptional set $E^{+}$of $f$.

Theorem 3. Let $f$ be a 1-stable meromorphic self map of a compact Kähler surface, with small topological degree, and let $T^{ \pm}=\omega^{ \pm}+d d^{c} G^{ \pm}$be the invariant currents. Suppose that $G^{+}$is finite at each point of $I^{-}$and $G^{-}$is finite at each point in $\mathrm{I}^{+}$. Assume furthermore that

- either $T^{+} \in L^{1}\left(T^{-}\right)$,

- or no point in either $I^{+}$or $I^{-}$is spurious.

Then $T^{+} \in \mathcal{E}\left(T^{-}\right)$and $T^{-} \in \mathcal{E}\left(T^{+}\right)$with weight $\chi(t)=t$.

We do not define "spurious" here (see §3), but we do note that the second assumption is satisfied when, for instance, the classes of $T^{+}$and $T^{-}$are Kähler. Also, when $\lambda_{2}=1$ it holds up to a birational change of surface [BD], Proposition 4.1. Finiteness of $G^{ \pm}$on $I^{\mp}$ is a kind of "avoidance" condition on the orbits of $I^{+}$and $I^{-}$that is readily verified for many maps.

We show in Section 4 that the hypotheses of Theorem 3 our satisfied in particular for

- polynomial maps of $\mathbb{C}^{2}$,

- maps on $\mathbb{P}^{1} \times \mathbb{P}^{1}$ that come from the secant algorithm for finding roots of polynomials,

- maps on surfaces $X$ with $\operatorname{kod}(X)=0$.

We also give weaker "indeterminacy avoidance" conditions that guarantee finite energy with respect to the other homogeneous weights $\chi(t)=-(-t)^{p}$. By studying 
a birational example of Favre, whose salient feature is an invariant line on which the map $f$ acts by rotation, we show how it can happen that the weaker avoidance conditions are verified when the stronger ones are not. For birational mappings with $T^{+} \in L^{1}\left(T^{-}\right)$it was proven in [DG] that

$$
\text { finite energy with respect to the weight } \chi(t)=t
$$

is equivalent to

$$
\log d\left(\cdot, I^{+}\right) \in L^{1}\left(\mu_{f}\right) .
$$

On the other hand, it follows from Theorem 2 and the results of [Du2] that, if a birational map $f$ with $\lambda_{1}>1$ has finite dynamical energy then it enjoys many interesting dynamical properties. Thus, the examples in $\S 4.4$ show that for birational maps, it is possible that the results of [Du2] hold while $\log d\left(\cdot, I^{+}\right) \notin L^{1}\left(\mu_{f}\right)$.

Less formally but more suggestively, our methods allow us to prove that $\mu_{f}$ acts much like a non-uniformly hyperbolic measure even though $\mu_{f}$ does not satisfy a convenient hypothesis guaranteeing existence of well-defined Lyapunov exponents (see e.g. Section S.2 in [KaHa] for background on these).

It is worth emphasizing that we do not know any example violating the finite dynamical energy condition. ${ }^{1}$ A rational map with small topological degree and infinite dynamical energy will tend to have some very surprising properties. For instance, we obtain the following dichotomy:

Theorem 4. Let $f$ be a 1-stable meromorphic self map of a compact Kähler surface, with small topological degree. Assume further that $T^{+} \in L^{1}\left(T^{-}\right)$. Then

- either $f$ has finite dynamical energy,

- or $\mu_{f}=T^{+} \wedge T^{-}$charges the pluripolar set $\left\{G^{+}+G^{-}=-\infty\right\}$.

The family of birational maps given in Example 4.7 satisfies this dichotomy. Furthermore, generic members of this family indeed have finite dynamical energy.

The structure of the paper is as follows. In $§ 1$, we develop the general framework for intersection of positive closed $(1,1)$ currents satisfying the finite energy condition. Theorem 1 and a general dichotomy leading to Theorem 4 are both established here. In $\S 2$, we consider the nature of the measure $\mu_{f}$ under the assumption that $f$ has finite dynamical energy. Theorem 2 is the end result. In $\S 3$, we consider the particular case of homogeneous weights more carefully, giving several criteria for finite dynamical energy along the lines of Theorem 3. Finally, in $\S 4$, we illustrate our main results by applying them to several significant examples, both invertible and non-invertible ones among them.

Acknowledgment. We would like to thank A. Zeriahi for several useful discussions.

\footnotetext{
${ }^{1}$ Added in proof: such examples have been very recently constructed by X. Buff [Bu].
} 


\section{Weighted energy with respect to a current}

We develop in this section the pluripotential theoretic tools that we will use to define and understand the wedge product $T^{+} \wedge T^{-}$. There is no dynamics here, so these results might accordingly be of independent interest.

1.1. The class $\boldsymbol{E}(\boldsymbol{T}, \boldsymbol{\alpha})$. Throughout the section, we take $\alpha, T$ to be positive closed $(1,1)$ currents on $X$ such that $\alpha$ has bounded potentials. We assume $\int_{X} \alpha \wedge T=$ $\{\alpha\} \cdot\{T\}=1$, the middle term denoting intersection of cohomology classes. We let $\operatorname{PSH}(X, \alpha)=\left\{u \in L^{1}(X): \alpha+d d^{c} u \geq 0\right\}$ denote the set of $\alpha$-plurisubharmonic (or just $\alpha$-psh) functions. If $u \in \operatorname{PSH}(X, \alpha)$ for $\alpha$ smooth, then $u$ is called quasiplurisubharmonic (or just qpsh).

If $u, v \in \operatorname{PSH}(X, \alpha)$ are bounded, it follows from plurifine considerations (see [BT2]) that

$$
\mathbf{1}_{\{u>v\}}\left[\alpha+d d^{c} u\right] \wedge T=\mathbf{1}_{\{u>v\}}\left[\alpha+d d^{c} \max (u, v)\right] \wedge T
$$

in the sense of Borel measures.

Let $\varphi \in \operatorname{PSH}(X, \alpha)$ be an unbounded function and let $\varphi_{j}:=\max (\varphi,-j) \in$ $\operatorname{PSH}(X, \alpha) \cap L^{\infty}(X)$ be its canonical approximants. We have $\varphi_{j} \searrow \varphi$, and by (3),

$$
\mathbf{1}_{\{\varphi>-k\}}\left[\alpha+d d^{c} \varphi_{j}\right] \wedge T=\mathbf{1}_{\{\varphi>-k\}}\left[\alpha+d d^{c} \varphi_{k}\right] \wedge T
$$

whenever $j \geq k$, since $\left\{\varphi_{j}>-k\right\}=\{\varphi>-k\}$ and $\max \left(\varphi_{j},-k\right)=\varphi_{k}$. Observe that $\{\varphi>-k\} \subset\{\varphi>-j\}$. Hence

$$
\mu_{j}(\varphi, T):=\mathbf{1}_{\{\varphi>-j\}}\left[\alpha+d d^{c} \varphi_{j}\right] \wedge T
$$

is an increasing sequence of Borel measures on $X$, whose total mass is bounded from above by $1=\{\alpha\} \cdot\{T\}$.

Definition 1.1. We set $\mu(\varphi, T):=\lim \nearrow \mu_{j}(\varphi, T)$ and

$$
\mathcal{E}(T, \alpha):=\{\varphi \in \operatorname{PSH}(X, \alpha) / \mu(\varphi, T)(X)=1\} .
$$

Alternatively $\varphi \in \mathcal{E}(T, \alpha)$ if and only if $\left[\alpha+d d^{c} \varphi_{j}\right] \wedge T(\{\varphi \leq-j\}) \rightarrow 0$.

Observe that the probability measures $\left[\alpha+d d^{c} \varphi_{j}\right] \wedge T$ converge to $\mu(\varphi, T)$ as Borel measures (i.e. in mass) when $\varphi \in \mathcal{E}(T, \alpha)$. This is much stronger than convergence as Radon measures (i.e. in the weak topology) and furnishes the key to the next proposition.

Proposition 1.2. Assume $\varphi \in \mathcal{E}(T, \alpha)$. Then

(1) the measures $\alpha \wedge T$ and $\mu(\varphi, T)$ do not charge the set $\{\varphi=-\infty\}$; 
(2) if $T$ puts no mass on a complete pluripolar set $P$, then neither does $\mu(\varphi, T)$.

Proof. We can suppose that $\varphi \leq 0$. To simplify notation we set $\mu=\mu(\varphi, T)$ and $\mu_{j}=\mu_{j}(\varphi, T)$. Note first that $\mu_{j}(\{\varphi=-\infty\})=0$, and hence $\mu(\{\varphi=-\infty\})=0$.

Fix $\chi: \mathbb{R} \rightarrow \mathbb{R}$ a convex increasing function such that $\chi(-\infty)=-\infty$ and $\chi \circ \varphi \in L^{1}(\mu)$ (see Lemma 1.3 below). Since $0 \geq \varphi_{j} \geq \varphi$ and $0 \leq \mu_{j} \leq \mu$, we have

$$
\sup _{j} \int_{X}(-\chi) \circ \varphi_{j} d \mu_{j} \leq \int_{X}(-\chi) \circ \varphi d \mu<+\infty .
$$

By Stokes' theorem,

$$
\int_{X}(-\chi) \circ \varphi_{j}\left(\alpha+d d^{c} \varphi_{j}\right) \wedge T=\int(-\chi) \circ \varphi_{j} \alpha \wedge T+\int \chi^{\prime} \circ \varphi_{j} d \varphi_{j} \wedge d^{c} \varphi_{j} \wedge T,
$$

where the rightmost term is non-negative. Observe that the measures $\left(\alpha+d d^{c} \varphi_{j}\right) \wedge T$ and $\mu$ have the same mass since $\varphi \in \mathcal{E}(T, \alpha)$ and coincide in $(\varphi>-j)$, so

$$
\left(\alpha+d d^{c} \varphi_{j}\right) \wedge T(\varphi \leq-j)=\mu(\varphi \leq-j) .
$$

Therefore

$\int_{(\varphi \leq-j)}(-\chi) \circ \varphi_{j}\left(\alpha+d d^{c} \varphi_{j}\right) \wedge T=(-\chi)(-j) \mu(\varphi \leq-j) \leq \int_{(\varphi \leq-j)}(-\chi) \circ \varphi d \mu$, which yields

$$
\int_{X}(-\chi) \circ \varphi_{j} \alpha \wedge T \leq \int_{X}(-\chi) \circ \varphi d \mu<+\infty .
$$

We infer $\chi \circ \varphi \in L^{1}(\alpha \wedge T)$, hence in particular $\alpha \wedge T(\varphi=-\infty)=0$.

Let $P$ be a complete pluripolar set, i.e. $P=\{\psi=-\infty\}$ for some quasiplurisubharmonic function $\psi \leq 0$ on $X$. Let $\omega$ be a Kähler form. By assumption $\omega \wedge T(P)=0$. Attenuating the singularities of $\psi$ if necessary (i.e. replacing $\psi$ by $\chi \circ \psi$ for some convex increasing function $\chi$ with slow growth at $-\infty$ and such that still $\chi(-\infty)=-\infty)$, we can assume $\psi \in L^{1}(\omega \wedge T)$.

Write $\alpha=\theta+d d^{c} u$, where $\theta$ is smooth and $u$ is bounded. We can also assume without loss of generality that $\omega^{\prime}=\theta+\omega$ is Kähler and $d d^{c} \psi \geq-\omega^{\prime}$ (replace $\omega$ by $A \omega, A \gg 1$, if necessary).

We claim that for every bounded $\alpha$-psh function $v, \psi \in L^{1}\left(\left[\alpha+d d^{c} v\right] \wedge T\right)$. Indeed

$$
0 \leq \int(-\psi)\left[\alpha+d d^{c} v\right] \wedge T \leq \int(-\psi) \omega^{\prime} \wedge T+\int(u+v)\left(-d d^{c} \psi\right) \wedge T .
$$

Now we can assume that $u+v \geq 0$ since these functions are bounded. The conclusion follows by observing that $-d d^{c} \psi \leq \omega^{\prime}$.

This shows in particular that $\mu_{j}=\left[\alpha+d d^{c} \varphi_{j}\right] \wedge T(P)=0$, hence $\mu(P)=0$ since $\left(\mu_{j}\right)$ converges to $\mu$ in the strong sense of Borel measures. 
We now introduce a set of weights.

Notation. We let $\mathcal{W}$ be the set of all convex increasing functions $\chi: \mathbb{R} \rightarrow \mathbb{R}$ such that $\chi(-\infty)=-\infty$ and $\chi(0)=0$.

A straightforward computation (see [GZ2]) shows that if $\varphi \in \operatorname{PSH}(X, \alpha)$ and $\chi^{\prime} \circ \varphi \leq 1$, then $\chi \circ \varphi \in \operatorname{PSH}(X, \alpha)$.

Lemma 1.3. Let $\mu$ be a positive measure and $u$ a measurable function which is bounded from above and such that $u>-\infty \mu$-a.e. Then there exists $\chi \in \mathcal{W}$ such that $\chi \circ u \in L^{1}(\mu)$.

Proof. Assume for simplicity that $u$ is negative. From the identity

$$
\int(\chi \circ u) d \mu=\int_{0}^{\infty} \mu(\chi \circ u<-t) d t
$$

it is straightforward to construct a piecewise affine $\chi$ that suffices.

Proposition 1.4. Assume that $T$ has the form $T=\beta+d d^{c} \psi$, with $\beta$ a positive current with bounded potentials, and $T$ puts no mass on the set $\{\psi=-\infty\}$. Then $T$ does not charge pluripolar sets.

Consequently if $\varphi \in \mathcal{E}(T, \alpha)$, then $\mu(\varphi, T)$ does not charge pluripolar sets.

Proof. Fix a Kähler form $\omega$. Replacing $\beta$ with $\beta+\omega$, we may assume $\{\beta\}^{2}>0$. If $P \subset X$ is locally pluripolar then by Theorem 7.2 in [GZ1], $P \subset\{u=-\infty\}$ for some $u \in \operatorname{PSH}(X, \beta)$. Fix $\chi \in \mathcal{W}$ such that $\chi \circ \psi \in L^{1}(T \wedge \omega)$.

We can assume without loss of generality that $\chi \circ u \in \operatorname{PSH}(X, \beta)$ and $\int_{X}(-\chi) \circ$ $u\left[\beta+d d^{c} u\right] \wedge \omega<+\infty$, replacing $u$ by $\chi \circ(u-C), C>0$, if necessary (in other words it is no loss of generality to assume $u$ has "small singularities", see [CGZ] for more detail). The comparison principle (see Proposition 2.5 in [GZ2]) then gives

$$
\int(-\chi) \circ u T \wedge \omega \leq 2 \int(-\chi) \circ u\left[\beta+d d^{c} u\right] \wedge \omega+2 \int(-\chi) \circ \psi T \wedge \omega<+\infty \text {. }
$$

Therefore $\chi \circ u \in L^{1}(T \wedge \omega)$, in particular $T \wedge \omega(P) \leq T \wedge \omega(\{u=-\infty\})=0$.

The second statement of the proposition follows from Proposition 1.2.

We introduce another class of $\alpha$-psh functions.

Definition 1.5. For $\chi \in \mathcal{W}$ we set

$$
\varepsilon_{\chi}(T, \alpha):=\left\{\varphi \in \operatorname{PSH}(X, \alpha) / \sup _{j \in \mathbb{N}} \int_{X}(-\chi) \circ \varphi_{j}\left[\alpha+d d^{c} \varphi_{j}\right] \wedge T<+\infty\right\},
$$

where $\varphi_{j}:=\max (\varphi,-j)$ are the canonical approximants. 
The relationship between these classes and $\mathscr{E}(T, \alpha)$ is the following.

Proposition 1.6. We have

$$
\varepsilon(T, \alpha)=\bigcup_{\chi \in \mathcal{W}} \varepsilon_{\chi}(T, \alpha)
$$

Proof. Given $\varphi \in \mathcal{E}_{\chi}(T, \alpha)$, we may assume $\varphi \leq 0$. Then

$0 \leq\left[\alpha+d d^{c} \varphi_{j}\right] \wedge T(\{\varphi \leq-j\}) \leq \frac{1}{|\chi(-j)|} \sup _{k} \int_{X}\left(-\chi \circ \varphi_{k}\right)\left[\alpha+d d^{c} \varphi_{k}\right] \wedge T \rightarrow 0$ since $\chi(-\infty)=-\infty$. Hence $\varphi \in \mathcal{E}(T, \alpha)$.

Conversely assume $\varphi \in \mathcal{E}(T, \alpha)$. By Proposition 1.2 and Lemma 1.3, there is weight $\chi \in \mathcal{W}$ such that $\chi \circ \varphi \in L^{1}(\mu(\varphi, T))$. We have by definition

$$
\mu(\varphi, T)(\{\varphi>-j\})=\left[\alpha+d d^{c} \varphi_{j}\right] \wedge T(\{\varphi>-j\})
$$

Since the measures on either side have the same total mass, we infer that

$$
\mu(\varphi, T)(\{\varphi \leq-j\})=\left[\alpha+d d^{c} \varphi_{j}\right] \wedge T(\{\varphi \leq-j\})
$$

Writing $\mu(\varphi)=\mu(\varphi, T)$ and $\alpha_{\varphi_{j}}:=\alpha+d d^{c} \varphi_{j}$, we use (4) again to get

$$
\begin{aligned}
\int_{X}\left(-\chi \circ \varphi_{j}\right) \alpha_{\varphi_{j}} \wedge T & =(-\chi)(-j) \int_{\{\varphi \leq-j\}} \alpha_{\varphi_{j}} \wedge T+\int_{\{\varphi>-j\}}(-\chi \circ \varphi) \alpha_{\varphi_{j}} \wedge T \\
& =(-\chi)(-j) \int_{\{\varphi \leq-j\}} d \mu(\varphi)+\int_{\{\varphi>-j\}}(-\chi \circ \varphi) d \mu(\varphi) \\
& \leq \int_{X}(-\chi \circ \varphi) d \mu(\varphi) .
\end{aligned}
$$

So $\varphi \in \varepsilon_{\chi}(T, \alpha)$.

1.2. Intersection of currents. Let $\alpha, \beta, S, T$ be positive closed $(1,1)$ currents on $X$ such that

(1) $\alpha, \beta$ have bounded potentials;

(2) $S=\alpha+d d^{c} \varphi$ for some $\varphi \in \operatorname{PSH}(X, \alpha)$;

(3) $T=\beta+d d^{c} \psi$ for some $\psi \in \operatorname{PSH}(X, \beta)$;

(4) $\{\alpha\} \cdot\{\beta\}=\int_{X} \alpha \wedge \beta=\{S\} \cdot\{T\}=1$. 
We want to define the wedge product $S \wedge T$. That is we want to construct a probability measure $\mu$ such that whenever $\varphi_{j} \in \operatorname{PSH}(X, \alpha) \cap L^{\infty}(X), \psi_{j} \in$ $\operatorname{PSH}(X, \beta) \cap L^{\infty}(X)$ decrease towards $\varphi, \psi$ then

$$
S_{j} \wedge T_{j}:=\left[\alpha+d d^{c} \varphi_{j}\right] \wedge\left[\beta \wedge d d^{c} \psi_{j}\right] \rightarrow \mu
$$

in the weak sense of Radon measures. Recall that the wedge product $S_{j} \wedge T_{j}$ of closed currents with bounded potentials is well defined thanks to the work of E. Bedford and A. Taylor [BT1]. It is well known that it is not always possible to define $S \wedge T$ when the currents have unbounded potentials, even if $S=T$. We show here that it is nevertheless possible in some very general situations.

The $L^{1}$ condition. When the potential $\varphi$ of $S$, is integrable with respect to (the trace measure of) the current $T$, then the current $\varphi T$ is well-defined, as is therefore

$$
\mu=S \wedge T:=\alpha \wedge T+d d^{c}(\varphi T) .
$$

It is well known that the continuity property $(\dagger)$ holds in this case. This is a consequence of the following lemma, which we will need for other purposes.

Lemma 1.7. Assume $\varphi \in L^{1}(T)$. Then for any bounded quasiplurisubharmonic function $u$, and for any sequence $\varphi_{j} \in \operatorname{PSH}(X, \alpha)$ decreasing to $\varphi$, one has $\varphi_{j} \in$ $L^{1}(T)$ and

$$
\int_{X} u\left[\alpha+d d^{c} \varphi_{j}\right] \wedge T \rightarrow \int_{X} u\left[\alpha+d d^{c} \varphi\right] \wedge T .
$$

Proof. By the monotone convergence theorem $\varphi_{j} T \rightarrow \varphi T$ as currents. Therefore $\left[\alpha+d d^{c} \varphi_{j}\right] \wedge T \rightarrow\left[\alpha+d d^{c} \varphi\right] \wedge T$ in the weak sense of Radon measures. Hence we are done if $u$ is continuous. Since $u$ is upper semi-continuous, we get that

$$
\limsup \int_{X} u\left[\alpha+d d^{c} \varphi_{j}\right] \wedge T \leq \int_{X} u\left[\alpha+d d^{c} \varphi\right] \wedge T .
$$

We now use the assumption that $u \in \operatorname{PSH}(X, \gamma)$ for some positive closed $(1,1)$ current $\gamma$ with bounded potentials. It follows from repeated application of Stokes' theorem that

$$
\begin{aligned}
\int_{X} u\left[\alpha+d d^{c} \varphi_{j}\right] \wedge T & =\int u \alpha \wedge T+\int_{X} \varphi_{j}\left[\gamma+d d^{c} u\right] \wedge T-\int_{X} \varphi_{j} \gamma \wedge T \\
& \geq \int_{X} u\left[\alpha+d d^{c} \varphi\right] \wedge T+\int_{X}\left(\varphi-\varphi_{j}\right) \gamma \wedge T .
\end{aligned}
$$

The integrations by parts are easily justified because $u$ is bounded. By monotone convergence, $\int_{X}\left(\varphi-\varphi_{j}\right) \gamma \wedge T \rightarrow 0$. So we infer

$$
\liminf \int_{X} u\left[\alpha+d d^{c} \varphi_{j}\right] \wedge T \geq \int_{X} u\left[\alpha+d d^{c} \varphi\right] \wedge T
$$


Note that the condition $\varphi \in L^{1}(T)$ is symmetric, $\varphi \in L^{1}(T) \Leftrightarrow \psi \in L^{1}(S)$. This follows from the Stokes theorem: if $\omega$ is any fixed Kähler form, then

$$
\int_{X} \varphi T \wedge \omega=\int_{X} \psi S \wedge \omega+\int_{X} \varphi \beta \wedge \omega-\int_{X} \psi \alpha \wedge \omega,
$$

where the last two integrals are finite because qpsh functions are always integrable with respect to measures of the form $\alpha \wedge \omega$ (resp. $\beta \wedge \omega$ ) (see e.g. [De]).

Lastly, note that if $\alpha_{1}+d d^{c} \varphi_{1}$ and $\alpha_{2}+d d^{c} \varphi_{2}$ are two representations of the same closed positive current $S$, with $\alpha_{i}$ of bounded potential, then $\varphi_{1} \in L^{1}(T)$ iff $\varphi_{2} \in L^{1}(T)$. It therefore makes sense to write " $S \in L^{1}(T)$ " as a shorthand for " $\varphi \in L^{1}(T)$ for some choice of $\alpha$ ". Thus we have just seen that

$$
S \in L^{1}(T) \Longleftrightarrow T \in L^{1}(S) .
$$

In the next paragraph we will give a different approach to the wedge product, using the energy formalism.

The energy condition. We will show that the wedge product $S \wedge T$ can be defined as $\mu(\varphi, T)$ whenever $\varphi \in \mathcal{E}(T, \alpha)$.

Proposition 1.8. Assume $\varphi \in L^{1}(T)$ so that the probability measure $S \wedge T$ is well defined. Then

$$
\mu(\varphi, T)=\mathbf{1}_{\{\varphi>-\infty\}} S \wedge T .
$$

Therefore $\varphi \in \mathcal{E}(T, \alpha)$ if and only if $S \wedge T(\{\varphi=-\infty\})=0$.

Proof. Without loss of generality, assume that $\varphi<0$. Let $\varphi_{j}:=\max (\varphi,-j)$ and $u_{k}:=\max (\varphi / k+1,0) \in \operatorname{PSH}(X, \alpha) \cap L^{\infty}(X)$, where $k \leq j$ is fixed. Observe that $\left\{u_{k}>0\right\}=\{\varphi>-k\}$ and $u_{k}=0$ elsewhere, thus

$$
u_{k}\left[\alpha+d d^{c} \varphi_{j}\right] \wedge T=u_{k} \mathbf{1}_{\{\varphi>-j\}}\left[\alpha+d d^{c} \varphi_{j}\right] \wedge T=u_{k} \mu_{j}(\varphi, T),
$$

Letting $j \rightarrow+\infty$ we infer, by using Lemma 1.7, that

$$
\left\langle u_{k} S \wedge T, h\right\rangle=\left\langle u_{k} \mu(\varphi, T), h\right\rangle, \quad \text { for all } k \in \mathbb{N}
$$

and for any continuous test function $h$ on $X$. Now $u_{k} \nearrow \mathbf{1}_{\{\varphi>-\infty\}}$, so

$$
\left\langle\mathbf{1}_{\{\varphi>-\infty\}} S \wedge T, h\right\rangle=\langle\mu(\varphi, T), h\rangle
$$

since $\mu(\varphi, T)$ does not charge $\{\varphi=-\infty\}$.

Proposition 1.8 implies that whenever $S \in L^{1}(T)$, one has $\varphi \in \mathcal{E}(T, \alpha)$ if and only if $\psi \in \mathcal{E}(S, \beta)$, and in either case $\mu(\varphi, T)=\mu(\psi, S)$. The next result gives some symmetry even without the integrability assumption. It follows from a monotone convergence argument that we defer until the next subsection. 
Theorem 1.9. Assume $\varphi \in \mathcal{E}(T, \alpha)$ and $\psi \in \mathcal{E}(S, \beta)$. Assume moreover that $T$ does not charge pluripolar sets. Then

$$
\mu(\varphi, T)=\mu(\psi, S)
$$

and this measure does not charge pluripolar sets.

If, moreover, $\varphi_{j} \in \operatorname{PSH}(X, \alpha), \psi_{j} \in \operatorname{PSH}(X, \beta)$ decrease to $\varphi, \psi$, and we set $S_{j}:=\alpha+d d^{c} \varphi_{j}, T_{j}:=\beta+d d^{c} \psi_{j}$, then we have $\varphi_{j} \in \mathcal{E}\left(T_{j}, \alpha\right), \psi_{j} \in \mathcal{E}\left(S_{j}, \beta\right)$ and

$$
\mu\left(\varphi_{j}, T_{j}\right)=\mu\left(\psi_{j}, S_{j}\right) \rightarrow \mu(\varphi, T)=\mu(\psi, S)
$$

in the weak sense of Radon measures.

Note that the condition $\varphi \in \mathcal{E}(T, \alpha)$ does not depend on the choice of $\alpha$.

Proposition 1.10. Assume that $S=\alpha_{1}+d d^{c} \varphi_{1}=\alpha_{2}+d d^{c} \varphi_{2}$, where $\alpha_{1}, \alpha_{2}$ have bounded potentials. Then $\mu\left(\varphi_{1}, T\right)=\mu\left(\varphi_{2}, T\right)$. In particular $\varphi_{1} \in \mathcal{E}\left(T, \alpha_{1}\right)$ if and only if $\varphi_{2} \in \mathcal{E}\left(T, \alpha_{2}\right)$.

Proof. Fix $u$ bounded such that $\alpha_{2}=\alpha_{1}+d d^{c} u$. Subtracting a constant if necessary, we can assume $\varphi_{1}=\varphi_{2}+u$. Fix $M>0$ such that $-M \leq u \leq+M$.

Observe that $\max \left(\varphi_{2},-j\right)+u=\max \left(\varphi_{1},-j+M\right)$ in the plurifine open set $\left\{\varphi_{1}>-j+M\right\} \subset\left\{\varphi_{2}>-j\right\}$. Thus

$$
\begin{aligned}
& \mathbf{1}_{\left\{\varphi_{1}>-j+M\right\}}\left[\alpha_{1}+d d^{c} \max \left(\varphi_{1},-j+M\right)\right] \wedge T \\
& \quad \leq \mathbf{1}_{\left\{\varphi_{2}>-j\right\}}\left[\alpha_{2}+d d^{c} \max \left(\varphi_{2},-j\right)\right] \wedge T \leq \mu\left(\varphi_{2}, T\right) .
\end{aligned}
$$

We infer $\mu\left(\varphi_{1}, T\right) \leq \mu\left(\varphi_{2}, T\right)$, whence equality by reversing the roles of $\varphi_{1}$ and $\varphi_{2}$. In particular $\varphi_{1} \in \mathcal{E}\left(T, \alpha_{1}\right)$ if and only if $\varphi_{2} \in \mathcal{E}\left(T, \alpha_{2}\right)$.

We already know that if both $\varphi_{j}$ and $\psi_{j}$ are bounded, then $\mu\left(\varphi_{j}, T_{j}\right)=S_{j} \wedge T_{j}$. Hence under the hypotheses of Theorem 1.9, our results now make it reasonable to set

$$
S \wedge T:=\mu(\varphi, T)=\mu(\psi, S) .
$$

Also we can write " $S \in \mathcal{E}(T)$ " to say that $\varphi \in \mathcal{E}(T, \alpha)$ for some choice of $\alpha$. In summary, we have shown that if $S \in \mathcal{E}(T)$ and $T \in \mathcal{E}(S)$, then there is a well-defined wedge product $S \wedge T$. We stress that our definition of $S \wedge T$ applies even in some cases where $S \notin L^{1}(T)$ (see [GZ2], §2.4). On the other hand, the case where $S$ and $T$ are transversely intersecting lines shows that $S \in L^{1}(T)$ does not mean that $S \in \varepsilon(T)$.

We now discuss the hypothesis made in Theorem 1.9 that $T$ does not charge pluripolar sets. Later on we will apply this construction to the case where $T=T^{+}$is the canonical $f^{*}$-invariant current associated to a 1-stable endomorphism $f: X \rightarrow$ $X$ of small topological degree. The laminar structure and extremality properties of $T^{+}$will allow us to reach the following alternative: 
- either $T^{+}$does not charge pluripolar sets,

- or $T^{+}$is supported on a complete pluripolar set.

It is therefore important to notice that the latter possibility can not occur under the finite energy assumption.

Proposition 1.11. Assume $T=\beta+d d^{c} \psi$ is supported on $(\psi=-\infty)$ and $\varphi \in$ $\mathcal{E}(T, \alpha)$. Then $\mu(\psi, S)=0$, hence in particular $\psi \notin \mathcal{E}(S, \beta)$.

Proof. Assume $\varphi \in \mathcal{E}(T, \alpha)$. It follows from Lemma 1.14 that $\varphi \in \mathcal{E}\left(T_{j}, \alpha\right)$, where $T_{j}=\beta+d d^{c} \psi_{j}, \psi_{j}=\max (\psi,-j)$.

Observe that by definition of $\mu\left(\varphi, T_{j}\right)$, the measures $T_{j} \wedge S_{k}$ converge (in the strong sense of Borel measures) towards $\mu\left(\varphi, T_{j}\right)$, as $k \rightarrow+\infty$. Here $S_{k}=\alpha+d d^{c} \varphi_{k}$, where $\varphi_{k}=\max (\varphi,-k)$.

Now $\mu\left(\varphi, T_{j}\right)=T_{j} \wedge S$, as follows from Proposition 1.8. We infer

$$
\mathbf{1}_{\{\psi>-j\}} T_{j} \wedge S_{k} \stackrel{k \rightarrow+\infty}{\longrightarrow} \mathbf{1}_{\{\psi>-j\}} T_{j} \wedge S .
$$

Observe finally that $\mathbf{1}_{\{\psi>-j\}} T_{j} \wedge S_{k}=\mathbf{1}_{\{\psi>-j\}} T \wedge S_{k}=0$ if $T$ is supported on $(\psi=$ $-\infty)$. The latter equality follows from lemma 1.12 below. Thus $\mathbf{1}_{\{\psi>-j\}} T_{j} \wedge S=0$, hence $\mu(\psi, S)=0$.

The following result is probably known to experts in pluripotential theory. Since we could not find a reference, we include a proof.

Lemma 1.12. Assume $T=d d^{c} \psi \geq 0$ is a positive closed $(1,1)$ current in the unit ball $\mathbf{B} \subset \mathbb{C}^{2}$, which gives full mass to $\{\psi=-\infty\}$. Then so does the measure $T \wedge d d^{c} u$, for any locally bounded plurisubharmonic function $u$.

Proof. This is a local question; we can assume all our objects are defined in a small neighborhood of $\overline{\mathbf{B}}$. Set $\varrho:=e^{\psi}$. This is a bounded psh function such that $\{\varrho>0\}=$ $\{\psi>-\infty\}$ and $\{\varrho=0\}=\{\psi=-\infty\}$. By assumption $\varrho T=0$ and we need to prove that $\varrho \mu=0$, where $\mu=T \wedge d d^{c} u, u \in \operatorname{PSH}(\mathbf{B}) \cap L_{\text {loc }}^{\infty}(\mathbf{B})$.

If $u$ is smooth, this easily follows from the identity

$$
\langle\varrho \mu, \chi\rangle=\left\langle\varrho T, \chi d d^{c} u\right\rangle
$$

valid for any test function $\chi$.

For the general case we approximate $u$ by a decreasing sequence of smooth psh functions $u_{j}$. Set $\mu_{j}:=T \wedge d d^{c} u_{j}$. Since

$$
\left\langle\varrho \mu_{j}, \chi\right\rangle=\left\langle\varrho T, \chi d d^{c} u_{j}\right\rangle=0,
$$


it suffices to show that the measures $\varrho \mu_{j}$ converge, in the weak sense of Radon measures, towards $\varrho \mu$. This is obvious if $\varrho$ is continuous. Let $\varrho_{\varepsilon}$ denote a sequence of smooth psh functions decreasing to $\varrho$. Since

$$
\lim _{j \rightarrow+\infty}\left\langle\varrho_{\varepsilon} \mu_{j}, \chi\right\rangle=\left\langle\varrho_{\varepsilon} \mu, \chi\right\rangle,
$$

for fixed $\varepsilon>0$, it suffices to show that $\varrho_{\varepsilon} \mu_{j} \rightarrow \varrho \mu_{j}$ as $\varepsilon \rightarrow 0$ uniformly with respect to $j$.

Using a "max-construction", we can assume without loss of generality that $\varrho_{\varepsilon} \equiv$ $\varrho \equiv u_{j} \equiv u \equiv\|z\|^{2}-1$ near $\partial \mathbf{B}$. This allows us to integrate by parts, since all these functions vanish on $\partial \mathbf{B}$. Let $\chi$ be a test function, then

$$
\begin{aligned}
& \left|\left\langle\varrho_{\varepsilon} \mu_{j}, \chi\right\rangle-\left\langle\varrho \mu_{j}, \chi\right\rangle\right| \\
& \quad \leq\|\chi\|_{e^{2}}\left\langle\left(\varrho_{\varepsilon}-\varrho\right) d d^{c} u_{j}, T\right\rangle \\
& \quad=C_{\chi} \int d\left(\varrho_{\varepsilon}-\varrho\right) \wedge d^{c} u_{j} \wedge T \\
& \quad \leq C_{\chi}\left(\int d\left(\varrho_{\varepsilon}-\varrho\right) \wedge d^{c}\left(\varrho_{\varepsilon}-\varrho\right) \wedge T\right)^{1 / 2}\left(\int d u_{j} \wedge d^{c} u_{j} \wedge T\right)^{1 / 2},
\end{aligned}
$$

as follows from Cauchy-Schwarz inequality.

The latter integral is uniformly bounded from above,

$$
\int d u_{j} \wedge d^{c} u_{j} \wedge T=\int\left(-u_{j}\right) \wedge d d^{c} u_{j} \wedge T \leq\|u\|_{L^{\infty}} \int d d^{c} u_{j} \wedge T \leq M_{0},
$$

while the next to last converges to zero,

$$
\int d\left(\varrho_{\varepsilon}-\varrho\right) \wedge d^{c}\left(\varrho_{\varepsilon}-\varrho\right) \wedge T \leq \int\left(\varrho_{\varepsilon}-\varrho\right) d d^{c} \varrho \wedge T \rightarrow 0,
$$

as follows from the monotone convergence theorem.

1.3. Proof of Theorem 1.9. We start with two useful inequalities.

Lemma 1.13. Fix $\chi \in W$ and let $u, v \in \operatorname{PSH}(X, \alpha) \cap L^{\infty}(X)$ be such that $u \leq v \leq 0$. Then

$$
0 \leq \int_{X}(-\chi \circ v)\left[\alpha+d d^{c} v\right] \wedge T \leq 2 \int_{X}(-\chi \circ u)\left[\alpha+d d^{c} u\right] \wedge T .
$$

The proof is a simple integration by parts (see Lemma 2.3 in [GZ2] for similar computation). It will follow from this lemma that in the definition of the class $\mathcal{E}_{\chi}(X, \alpha)$, one can replace the canonical approximants by any sequence of bounded $\alpha$-psh functions decreasing towards $\varphi$.

Fix $\gamma \geq 0$ a positive closed current with bounded potentials. For this lemma we use the notation $\alpha_{\varphi}:=\alpha+d d^{c} \varphi$ and $\gamma_{u}:=\gamma+d d^{c} u$. 
Lemma 1.14. Fix $\chi \in \mathcal{W}$ and $0 \geq \varphi \in \operatorname{PSH}(X, \alpha) \cap L^{\infty}(X)$. Let $u \leq v$ be two $\gamma$-psh functions. Then

$$
0 \leq \int_{X}(-\chi \circ \varphi) \alpha_{\varphi} \wedge \gamma_{v} \leq 2 \int_{X}(-\chi \circ \varphi) \alpha_{\varphi} \wedge \gamma_{u}+\chi^{\prime}(0) \int_{X}[v-u] \alpha^{2} .
$$

Proof. It follows from Stokes' theorem that

$$
\int(-\chi \circ \varphi) \alpha_{\varphi} \wedge \gamma_{v}=\int(-\chi \circ \varphi) \alpha_{\varphi} \wedge \gamma_{u}+\int(v-u) \alpha_{\varphi} \wedge\left[-d d^{c} \chi \circ \varphi\right] .
$$

Observe that $-d d^{c} \chi \circ \varphi \leq \chi^{\prime} \circ \varphi \alpha$, thus the latter integral is bounded from above by $I=\int(v-u) \chi^{\prime} \circ \varphi \alpha_{\varphi} \wedge \alpha$. Now

$$
\chi^{\prime} \circ \varphi \alpha_{\varphi} \leq \chi^{\prime} \circ \varphi \alpha_{\varphi}+\chi^{\prime \prime} \circ \varphi d \varphi \wedge d^{c} \varphi=\chi^{\prime} \circ \varphi \alpha+d d^{c}(\chi \circ \varphi),
$$

so we may estimate the integral $I$ :

$$
\begin{aligned}
I & \leq \int(-\chi \circ \varphi) \alpha \wedge d d^{c}(u-v)+\int(v-u) \chi^{\prime} \circ \varphi \alpha^{2} \\
& \leq \int(-\chi \circ \varphi) \alpha \wedge \gamma_{u}+\chi^{\prime}(0) \int(v-u) \alpha^{2} .
\end{aligned}
$$

The conclusion follows by observing that

$$
\int(-\chi \circ \varphi) \alpha_{\varphi} \wedge \gamma_{u}=\int(-\chi \circ \varphi) \alpha \wedge \gamma_{u}+\int \chi^{\prime} \circ \varphi d \varphi \wedge d^{c} \varphi \wedge \gamma_{u} \geq \int(-\chi \circ \varphi) \alpha \wedge \gamma_{u} .
$$

Proof of Theorem 1.9. Without loss of generality we can assume that $\varphi, \psi \leq 0$.

Step 1. Assume that $\varphi_{j}:=\max (\varphi,-j)$ and $\psi_{j}:=\max (\psi,-j)$ are the canonical approximants. We first show that the measures $S_{j} \wedge T_{j}$ converge to $\mu(\varphi, T)$. Recall that by definition,

$$
\mu(\varphi, T)=\lim \nearrow \mathbf{1}_{\{\varphi>-j\}} S_{j} \wedge T .
$$

Fix $N \in \mathbb{N}$. It follows from (3) that for all $j \geq N$,

$$
S_{j} \wedge T_{j} \geq \mathbf{1}_{\{\psi>-N\}} S_{j} \wedge T_{j}=\mathbf{1}_{\{\psi>-N\}} S_{j} \wedge T .
$$

Let $\sigma$ be a cluster point of the sequence $\left(S_{j} \wedge T_{j}\right)$. We infer

$$
\sigma \geq \lim _{N \rightarrow+\infty} \mathbf{1}_{\{\psi>-N\}} \mu(\varphi, T)=\mathbf{1}_{\{\psi>-\infty\}} \mu(\varphi, T)=\mu(\varphi, T),
$$

since $\mu(\varphi, T)$ does not charge the pluripolar set $\{\psi=-\infty\}$, as follows from Proposition 1.2 (because we assume $T$ does not charge pluripolar sets). Since both $\sigma$ and $\mu(\varphi, T)$ are probability measures, it follows that $\sigma=\mu(\varphi, T)$. 
We now show that $\mu(\varphi, T)=\mu(\psi, S)$. Observe first that

$$
\mathbf{1}_{\{\varphi>-j\} \cap\{\psi>-j\}} S_{j} \wedge T_{j} \rightarrow \mu(\varphi, T),
$$

where the convergence holds in the strong sense of Borel measures. Recall that $\mu(\psi, S)=\lim \nearrow \mathbf{1}_{\{\psi>-j\}} S \wedge T_{j}$. Now $\mathbf{1}_{\{\varphi>-j\}} S \wedge T_{j}=\mathbf{1}_{\{\varphi>-j\}} S_{j} \wedge T_{j}$, hence

$$
\mathbf{1}_{\{\varphi>-j\}}\left[\mathbf{1}_{\{\psi>-j\}} S \wedge T_{j}\right]=\mathbf{1}_{\{\varphi>-j\} \cap\{\psi>-j\}} S_{j} \wedge T_{j} .
$$

We infer $\mathbf{1}_{\{\varphi>-\infty\}} \mu(\psi, S)=\mu(\varphi, T)$. Since these are both probability measures, we conclude that $\mu(\psi, S)=\mu(\varphi, T)$ and this measure does not charge pluripolar sets (by Proposition 1.2).

Step 2. In the sequel we set $\mu=\mu(\varphi, T)=\mu(\psi, S)$ and we fix $\chi \in \mathcal{W}$ such that $\varphi \in \mathcal{E}_{\chi}(T, \alpha)$ and $\psi \in \mathcal{E}_{\chi}(S, \beta)$. We show that

$$
\chi \circ \varphi_{j} S_{j} \wedge T_{j} \rightarrow \chi \circ \varphi \mu
$$

in the strong sense of Borel measures.

Let $B$ be a Borel subset of $X$. We leave the reader check that $\int_{B} \chi \circ \varphi_{j} S_{j} \wedge T$ converges to $\int_{B} \chi \circ \varphi d \mu$ as $j \rightarrow \infty$. It then suffices to verify that $\int_{B} \chi \circ \varphi_{j} S_{j} \wedge$ $\left(T-T_{j}\right) \rightarrow 0$. It follows from (3) that

$$
\mathbf{1}_{\{\psi>-j\}} \chi \circ \varphi_{j} S_{j} \wedge T_{j}=\mathbf{1}_{\{\psi>-j\}} \chi \circ \varphi_{j} S_{j} \wedge T .
$$

Since

$$
\int_{B \cap\{\psi \leq-j\}}|\chi| \circ \varphi_{j} S_{j} \wedge T \leq \int_{\{\psi \leq-j\}}|\chi| \circ \varphi_{j} S_{j} \wedge T \rightarrow \int_{\{\psi=-\infty\}}|\chi| \circ \varphi d \mu=0,
$$

we will be done if we can show that $\int_{\{\psi \leq-j\}}|\chi| \circ \varphi_{j} S_{j} \wedge T_{j} \rightarrow 0$.

From Lemmas 1.13 and 1.14, we obtain $M_{\chi} \in \mathbb{R}$ such that

$$
\int(-\chi) \circ \varphi_{j} S_{j} \wedge T_{j} \leq M_{\chi}, \quad \int(-\chi) \circ \psi_{j} S_{j} \wedge T_{j} \leq M_{\chi} \quad \text { for all } j \in \mathbb{N} .
$$

Choose another weight $\tilde{\chi} \in W$, such that the same uniform bound hold, and such that moreover $\chi=o(\tilde{\chi})$ and $\chi / \tilde{\chi}$ is increasing (to find such a $\tilde{\chi}$, choose $\tilde{\chi}$ first and then $\chi$ !). We conclude that

$$
\int_{\{\psi \leq-j\}}|\chi| \circ \varphi_{j} S_{j} \wedge T_{j} \leq M_{\tilde{\chi}}\left|\frac{\chi}{\tilde{\chi}}(-j)\right| \rightarrow 0,
$$

as desired. 
From Step 2 we immediately obtain the following generalization of Lemma 1.13.

Corollary 1.15. Let $\chi \in W, u \in \mathcal{E}_{\chi}(T, \alpha)$ and $v \in \operatorname{PSH}(X, \alpha)$ such that $u \leq v \leq 0$. Then $v \in \mathcal{E}_{\chi}(T, \alpha)$ and

$$
0 \leq \int_{X}(-\chi \circ v)\left[\alpha+d d^{c} v\right] \wedge T \leq 2 \int_{X}(-\chi \circ u)\left[\alpha+d d^{c} u\right] \wedge T .
$$

Step 3. Let $\varphi_{j}, \psi_{j} \leq 0$ denote now arbitrary sequences of $\alpha$-psh, $\beta$-psh functions decreasing towards $\varphi, \psi$. From Lemma 1.14 and Corollary 1.15, we infer that $\varphi_{j} \in$ $\mathcal{E}_{\chi}\left(T_{j}, \alpha\right)$ and $\psi_{j} \in \mathcal{E}_{\chi}\left(S_{j}, \beta\right)$. Thus the measures $\mu\left(\varphi_{j}, T_{j}\right)=\mu\left(\psi_{j}, S_{j}\right)$ are well defined. We set

$$
\begin{aligned}
\varphi_{j}^{(K)}:=\max \left(\varphi_{j},-K\right), \quad \psi_{j}^{(K)}:=\max \left(\psi_{j},-K\right), \\
\varphi^{(K)}:=\max (\varphi,-K), \quad \psi^{(K)}:=\max (\psi,-K) .
\end{aligned}
$$

Similarly,

$$
\begin{array}{ll}
S_{j}^{(K)}:=\alpha+d d^{c} \varphi_{j}^{(K)}, & T_{j}^{(K)}:=\beta+d d^{c} \psi_{j}^{(K)}, \\
S^{(K)}:=\alpha+d d^{c} \varphi^{(K)}, & T^{(K)}:=\beta+d d^{c} \psi^{(K)} .
\end{array}
$$

It follows from Step 1 that $S^{(K)} \wedge T^{(K)} \rightarrow \mu$ and $S_{j}^{(K)} \wedge T_{j}^{(K)} \rightarrow S_{j} \wedge T_{j}$ when $K \rightarrow+\infty$. It follows from the monotone convergence theorem of [BT1] that for each fixed $K$,

$$
S_{j}^{(K)} \wedge T_{j}^{(K)} \rightarrow S^{(K)} \wedge T^{(K)} \text { as } j \rightarrow+\infty .
$$

Thus we will be done if we can prove that the convergence $S_{j}^{(K)} \wedge T_{j}^{(K)} \rightarrow S_{j} \wedge T_{j}$ is uniform with respect to $j$. This is what we show now.

Fix a Borel subset $B \subset X$. Note that $\left\{\varphi_{j} \leq-K\right\} \cup\left\{\psi_{j} \leq-K\right\} \subset\left\{u_{j} \leq-K\right\}$, where $u_{j}:=\varphi_{j}+\psi_{j}$. Fixing $\chi \in \mathcal{W}$ as at the end of Step 2, we have from convexity of $\chi$ that

$$
\sup _{j} \int_{X}\left(-\chi \circ u_{j}\right) S_{j} \wedge T_{j} \leq \sup _{j} \int_{X}\left(-\chi \circ \varphi_{j}\right) S_{j} \wedge T_{j}+\sup _{j} \int_{X}\left(-\chi \circ \psi_{j}\right) S_{j} \wedge T_{j} \leq 2 M_{\chi} \text {. }
$$

It follows again from (3) that $S_{j}^{(K)} \wedge T_{j}^{(K)} \equiv S_{j} \wedge T_{j}$ in the plurifine open set $\left\{\varphi_{j}>-K\right\} \cap\left\{\psi_{j}>-K\right\}$, thus

$$
\left|S_{j}^{(K)} \wedge T_{j}^{(K)}(B)-S_{j} \wedge T_{j}(B)\right| \leq \int_{\left\{u_{j} \leq-K\right\}}\left[S_{j}^{(K)} \wedge T_{j}^{(K)}+S_{j} \wedge T_{j}\right] .
$$


Lemma 1.14 and Corollary 1.15 imply that $\int(-\chi) \circ u_{j}^{(K)} S_{j}^{(K)} \wedge T_{j}^{(K)}$ is bounded above by $4 M_{\chi}+C$. Hence

$$
\left|S_{j}^{(K)} \wedge T_{j}^{(K)}(B)-S_{j} \wedge T_{j}(B)\right| \leq \frac{6 M_{\chi}+C}{|\chi(-K)|}
$$

converges to zero as $K \rightarrow+\infty$, uniformly with respect to $j$.

1.4. The gradient approach. We now give an alternative description of the finite energy conditions in terms of integrability properties of weighted gradients (in the spirit of [BD] who considered the special case $\chi(t)=t$ in what follows).

Recall that any function $\varphi \in \operatorname{PSH}(X, \alpha)$ has gradient in $L^{2-\varepsilon}(X)$ for any $\varepsilon>0$, but $\nabla \varphi \notin L^{2}(X)$ in general. Indeed, $\varphi$ has gradient in $L^{2}$ if and only if $\varphi \in L^{1}\left(\alpha_{\varphi}\right)$, where we write $\alpha_{\varphi}=\alpha+d d^{c} \varphi$ as before. More generally when $\alpha_{\varphi}:=\alpha+d d^{c} \varphi$ does not charge the set $\{\varphi=-\infty\}$, there exists $\chi \in \mathcal{W}$ such that $\chi \circ \varphi \in L^{1}\left(\alpha_{\varphi}\right)$. Hence

$$
\int_{X} \chi^{\prime} \circ \varphi d \varphi \wedge d^{c} \varphi \wedge \omega=\int_{X}(-\chi \circ \varphi) d d^{c} \varphi \wedge \omega \leq \int_{X}(-\chi \circ \varphi) \alpha_{\varphi} \wedge \omega<+\infty
$$

where $\omega$ is a fixed Kähler form. We get that $\varphi$ has weighted gradient in $L^{2}(X)$.

This suggests that we can give an alternative description of our energy conditions in terms of weighted gradients.

Definition 1.16. For $\chi \in \mathcal{W}$ we set

$$
\nabla_{\chi}(T, \alpha):=\left\{\varphi \in \operatorname{PSH}(X, \alpha) / \sup _{j \geq 0} \int_{X} \chi^{\prime} \circ \varphi_{j} d \varphi_{j} \wedge d^{c} \varphi_{j} \wedge T<+\infty\right\},
$$

where $\varphi_{j}:=\max (\varphi,-j)$ are the canonical approximants.

Proposition 1.17. Fix $\chi \in$ W. Then

$$
\mathcal{E}_{\chi}(T, \alpha)=\left\{\varphi \in \nabla_{\chi}(T, \alpha) / \chi \circ \varphi \in L^{1}(\alpha \wedge T)\right\}
$$

Proof. This follows from integrating by parts,

$$
\int\left(-\chi \circ \varphi_{j}\right) \alpha_{\varphi_{j}} \wedge T=\int\left(-\chi \circ \varphi_{j}\right) \alpha \wedge T+\int \chi^{\prime} \circ \varphi_{j} d \varphi_{j} \wedge d^{c} \varphi_{j} \wedge T .
$$

Remark 1.18. If $\chi^{\prime}$ is bounded above, then to verify the condition $\chi \circ \varphi \in L^{1}(\alpha \wedge T)$ in Proposition 1.17, it suffices to show simply that $\chi \circ \varphi \in L^{1}(T)$. 
Proof. Since $\alpha \geq 0$ has bounded potentials, we have $\alpha \leq c \omega+d d^{c} u$ where $u$ is bounded and, without loss of generality, positive. Thus,

$$
0 \leq \int-\chi \circ \varphi \alpha \wedge T \leq c \int-\chi \circ \varphi \omega \wedge T+\int-u d d^{c}(\chi \circ \varphi) \wedge T .
$$

The assertion therefore follows from the bound $-u d d^{c}(\chi \circ \varphi) \leq-u\left(\chi^{\prime} \circ \varphi\right) d d^{c} \varphi \leq$ $\left\|u \chi^{\prime}\right\|_{\infty} \omega^{+}$.

1.5. Examples. We give here simple criteria which ensure that some of our energy conditions are satisfied. We let $\mathcal{E}^{1}(T, \alpha)$ denote the class $\mathcal{E}_{\chi}(T, \alpha)$ for the weight $\chi(t)=t$. Observe that

$$
\mathcal{E}^{1}(T, \alpha)=\bigcap_{\chi \in \mathcal{W}} \mathcal{E}_{\chi}(T, \alpha)
$$

Proposition 1.19. If $\varphi \in \operatorname{PSH}(X, \alpha)$ is bounded, then $\varphi \in \mathcal{E}^{1}(T, \alpha)$.

This is easy and follows directly from the definitions. In dynamical situations, invariant currents with bounded potentials appear for instance for meromorphic maps on surfaces of Kodaira dimension zero (see $\S 4$ ). The next result is a bit more elaborate and will be useful in particular with $\varphi=\Gamma^{ \pm}$in $\S 3$.

Proposition 1.20. Assume $\varphi \in \operatorname{PSH}(X, \alpha) \cap L_{\mathrm{loc}}^{\infty}(X \backslash F)$, where $F$ is a finite set of points. Then $\varphi \in L^{1}(T)$.

Moreover if $\varphi \geq A \log \operatorname{dist}(\cdot, F)-A$ for some constant $A>0$, and if $v(T, p)=0$ for all $p \in F$, then $\varphi \in \mathcal{E}(T, \alpha)$.

Proof. We can assume without loss of generality that $\varphi \leq 0$ on $X$. Fix $\omega$ a Kähler form on $X$. We need to show that $\int_{X}(-\varphi) T \wedge \omega<+\infty$. Let $\omega^{\prime}$ be a smooth form cohomologous to $\omega$ which vanishes in a small neighborhood of $F$. We can find $h \geq 0$ a smooth function such that $\omega=\omega^{\prime}+d d^{c} h$. Now

$$
\begin{aligned}
\int_{X}(-\varphi) T \wedge \omega & =\int_{X}(-\varphi) T \wedge \omega^{\prime}+\int_{X} h T \wedge\left(-d d^{c} \varphi\right) \\
& \leq \int_{X}(-\varphi) T \wedge \omega^{\prime}+\int_{X} h T \wedge \alpha<+\infty,
\end{aligned}
$$

since $\varphi$ is bounded on the support of $T \wedge \omega^{\prime}$. We have used here that $h T \geq 0$ while $-d d^{c} \varphi \leq \alpha$. This shows that $\varphi \in L^{1}(T)$. Thus the measure $\mu:=S \wedge T=$ $\left(\alpha+d d^{c} \varphi\right) \wedge T$ is well defined.

Assume now that $\varphi \geq g:=A \log \operatorname{dist}(\cdot, F)-A$ for some constant $A>0$ and $v(T, p)=0$ for all $p \in F$. Assume $g \in \operatorname{PSH}(X, \alpha)$. Observe that $g \in L^{1}(T)$. Hence $\theta=\left[\alpha+d d^{c} g\right] \wedge T$ is a well defined positive measure which looks, locally 
near each point in $F$, like the projective mass of $T$. Since $v(T, p)=0$ when $p \in F$, we infer that $\theta(p)=0$. Therefore $\theta(\{g=-\infty\})=0$, and there exists $\chi \in \mathcal{W}$ such that $g \in \mathcal{E}_{\chi}(T, \alpha)$. It now follows from Corollary 1.15 that $\varphi \in \mathcal{E}_{\chi}(T, \alpha)$ also, since $\varphi$ is less singular than $g$.

When $g$ is not $\alpha$-psh, it is $\omega$-psh and hence $(\omega+\alpha)$-psh, for some Kähler form $\omega$. Observe that $\varphi$ is also $(\omega+\alpha)$-psh. We claim that $\varphi \in \mathcal{E}(T, \alpha)$ if and only if $\varphi \in \mathcal{E}(T, \alpha+\omega)$. Indeed

$$
\begin{aligned}
\mu(\varphi, T, \alpha+\{\omega\}) & =\lim _{j \rightarrow+\infty}\left[\mu_{j}(\varphi, T, \alpha)+\mathbf{1}_{\{\varphi>-j\}} \omega \wedge T\right] \\
& =\mu(\varphi, T, \alpha)+\mathbf{1}_{\{\varphi>-\infty\}} \omega \wedge T .
\end{aligned}
$$

Now $\omega \wedge T(\varphi=-\infty)=0$ since $(\varphi=-\infty) \subset F$ is finite. Thus

$\mathbf{M}(\mu(\varphi, T, \alpha+\{\omega\}))=\{T\} \cdot(\alpha+\{\omega\})$ if and only if $\mathbf{M}(\mu(\varphi, T, \alpha))=\{T\} \cdot \alpha$,

which is the desired result. Here $\mathbf{M}(\mu)$ denotes the total mass of the measure $\mu$. We can now conclude the proof by replacing $\alpha$ by $\alpha+\omega$ in the above argument.

\section{The canonical invariant measure}

Now let us return to the dynamical situation described in the introduction. For the remainder of this paper, $f: X \rightarrow X$ is a meromorphic transformation of a compact Kähler surface $(X, \omega)$. We always assume that

- $f$ is 1-stable, i.e. $\left(f^{n}\right)^{*}=\left(f^{*}\right)^{n}$ on $H^{1,1}(X, \mathbb{R})$ for all $n \in \mathbb{N}$;

- the dynamical degrees of $f$ satisfy $1 \leq \lambda_{2}(f)<\lambda_{1}:=\lambda_{1}(f)$.

With these conditions, our work in [DDG1] shows that the canonical current $T^{+}=\lambda_{1}^{-1} f^{*} T^{+}$in (1) exists and can be alternatively expressed

$$
T^{+}=\lim _{n \rightarrow \infty} \lambda_{1}^{-n} f^{n *} \omega^{+}=\omega^{+}+d d^{c} G^{+}, \quad \text { with } G^{+}=\sum_{n=0}^{\infty} \frac{\Gamma^{+} \circ f^{n}}{\lambda_{1}^{n}},
$$

where $\omega^{+}$is a positive closed current with bounded potentials cohomologous to $T^{+}$ and $d d^{c} \Gamma^{+}=\lambda_{1}^{-1} f^{*} \omega^{+}-\omega^{+}$. The canonical forward invariant current $T^{-}=$ $\lambda_{1}^{-1} f_{*} T^{-}$also exists and admits a similar description

$$
T^{-}=\lim _{n \rightarrow \infty} \lambda_{1}^{-n} f_{*}^{n} \omega^{-}=\omega^{-}+d d^{c} G^{-}, \quad \text { with } G^{-}=\sum_{n=0}^{\infty} \lambda_{1}^{-n} f_{*}^{n} \Gamma^{-} .
$$


2.1. The dynamical energy. We can assume, without loss of generality, that $\left\{\omega^{+}\right\}$. $\left\{\omega^{-}\right\}=\left\{T^{+}\right\} \cdot\left\{T^{-}\right\}=1$. Our aim here is to use the techniques from $\S 1$ to define a probability measure $\mu_{f}=T^{+} \wedge T^{-}$and understand its geometric and dynamical properties.

Definition 2.1. We say that $f$ has finite dynamical energy if

$$
T^{+} \in \mathcal{E}\left(T^{-}\right) \text {and } T^{-} \in \mathcal{E}\left(T^{+}\right) .
$$

We assume henceforth that our maps always have finite dynamical energy. Finite dynamical energy is equivalent via Proposition 1.6 to having $\chi \in W$ such that

$$
G^{+} \in \mathcal{E}_{\chi}\left(T^{-}, \omega^{+}\right) \text {and } G^{-} \in \mathcal{E}_{\chi}\left(T^{+}, \omega^{-}\right) .
$$

We say then more specifically that $f$ satisfies condition $\left(E_{\chi}\right)$. We shall see in $\S 4$ some weights $\chi \in \mathcal{W}$ that actually arise in specific families of examples.

The existence of $\mu_{f}$, in the sense of Theorem 1.9, is a consequence of finite dynamical energy. When $\operatorname{kod}(X)=0$, the invariant current $T^{-}$has bounded potentials, hence it does not charge pluripolar sets. When $X$ is rational, we instead consider $T^{+}$.

Proposition 2.2. Assume $X$ is rational and $f$ has finite dynamical energy. Then $T^{+}$ does not charge pluripolar sets.

Proof. The proof consists in establishing the following more precise alternative:

- either $T^{+}$does not charge pluripolar sets,

- or it is supported on a pluripolar set, hence $f$ cannot have finite dynamical energy.

Decompose $T^{+}$as $T^{+}=T_{n p}^{+}+T_{p p}^{+}$, where $T_{n p}^{+}=\mathbf{1}_{\left\{G^{+}>-\infty\right\}} T^{+}$does not charge pluripolar sets, while $T_{p p}^{+}=\mathbf{1}_{\left(G^{+}=-\infty\right)}$ gives full mass the pluripolar set $G^{+}=-\infty$. Because $T^{+}$is a strongly approximable laminar current (see [DDG1]) It follows from [Du1], Theorem 6.8, that this decomposition is closed, i.e. $T_{p p}^{+}, T_{n p}^{+}$are closed currents.

Since $T^{+}$is invariant (under $f^{*} / \lambda_{1}$ ) and does not charge (critical) curves (see Theorem 2.4 in [DDG1]), we also infer that $T_{p p}^{+}, T_{n p}^{+}$are both invariant. Now $T^{+}$is an extremal point of the cone of positive closed invariant currents (see Remark 2.2 in [DDG1]). Thus it follows that either $T^{+}=T_{n p}^{+}$does not charge pluripolar sets (see Proposition 1.4), or $T^{+}=T_{p p}^{+}$is supported on the pluripolar set $\left\{G^{+}=-\infty\right\}$. In the latter case, it follows from Proposition 1.11 that $f$ cannot have finite dynamical energy.

As it will be seen below and in [DDG3], the finite dynamical energy condition will allow us to understand the dynamics of $\mu_{f}$ quite thoroughly. From Proposition 1.8 we get the following nice alternative, which emphasizes the naturality of this assumption. 
Theorem 2.3. Assume that $T^{+} \in L^{1}\left(T^{-}\right)$, so that $\mu_{f}=T^{+} \wedge T^{-}$is well defined. Then

- either $\mu_{f}$ charges the pluripolar set $\left\{G^{+}=-\infty\right\} \cup\left\{G^{-}=-\infty\right\}$,

- or $f$ has finite dynamical energy.

\subsection{Mixing}

Theorem 2.4. Assume $f$ has finite dynamical energy. Then the measure $\mu_{f}=$ $T^{+} \wedge T^{-}$is $f$-invariant and mixing, and it does not charge pluripolar sets.

Proof. It follows from Proposition 2.2 and Theorem 1.9 that $\mu_{f}=T^{+} \wedge T^{-}$is a well defined probability measure which does not charge pluripolar sets. In particular, $\mu_{f}=T^{+} \wedge T^{-}$does not charge the pluripolar set $I^{+} \subset\left\{G^{+}=-\infty\right\}$. Moreover by Theorem 1.9,

$$
\mu_{f}=\lim _{n, p \rightarrow+\infty} \mu_{n, p}, \quad \text { where } \mu_{n, p}:=\frac{1}{\lambda_{1}^{n}}\left(f^{n}\right)^{*} \omega^{+} \wedge \frac{1}{\lambda_{1}^{p}}\left(f^{p}\right)_{*} \omega^{-} .
$$

Since $f_{*} \mu_{n, p}=\mu_{n-1, p+1}$ and since the operator $f_{*}$ is continuous on the set of probability measures which do not charge the indeterminacy set $I^{+}$, we infer $f_{*} \mu_{f}=$ $\mu_{f}$, i.e. $\mu_{f}$ is an invariant probability measure.

We now show that $\mu_{f}$ is mixing. Let $h$ and $k$ be test functions on $X$. We need to show that

$$
\int_{X} k h \circ f^{n} d \mu_{f} \rightarrow \int_{X} h d \mu_{f} \int_{X} k d \mu_{f} .
$$

Set $\mu_{j}:=\left[\omega^{-}+d d^{c} v_{j}\right] \wedge T^{+}$, where $v_{j}:=\max \left(G^{-},-j\right)$ are the canonical approximants of $G^{-}$. Fix $\chi \in \mathcal{W}$ such that $G^{-} \in \mathcal{E}_{\chi}\left(T^{+}, \omega^{-}\right)$. It follows from Corollary 1.15 that $\chi \circ G^{-} \in L^{1}\left(\mu_{f}\right), \chi \circ v_{j} \in L^{1}\left(\mu_{j}\right)$ uniformly in $j$, and also that $\mu_{j} \equiv \mu_{f}$ in the plurifine open set $\left\{G^{-}>-j\right\}$. Therefore

$$
\left|\int_{X} k h \circ f^{n} d \mu_{f}-\int_{X} k h \circ f^{n} d \mu_{j}\right| \leq\|k\|_{L^{\infty}}\|h\|_{L^{\infty}}\left(\mu_{f}+\mu_{j}\right)\left\{G^{-} \leq-j\right\}
$$

converges to zero as $j \rightarrow+\infty$, uniformly with respect to $n \in \mathbb{N}$.

It suffices then to replace $\mu_{f}$ by $\left(\omega^{-}+d d^{c} v\right) \wedge T^{+}$, where $v$ is a bounded $\omega^{-}$-psh function and show

$$
\int_{X} k h \circ f^{n}\left(\omega^{-}+d d^{c} v\right) \wedge T^{+} \rightarrow\left(\int k\left(\omega^{-}+d d^{c} v\right) \wedge T^{+}\right)\left(\int h d \mu_{f}\right) .
$$

Observe that here we can replace $\omega^{-}$by $\theta^{-}$, which is smooth. So it suffices to show the convergence for an arbitrary Kähler form $\omega$, instead of $\theta^{-}$. 
If $v$ is smooth the convergence follows from Proposition 2.5 below. For the general case, recall that we can approximate $v$ from above by a decreasing family of smooth $\omega$-psh functions $v_{\varepsilon}$. The proof will be finished if we show that $\int_{X} k h \circ$ $f^{n} d d^{c}\left(v_{\varepsilon}-v\right) \wedge T^{+}$converges to zero as $\varepsilon \rightarrow 0$, uniformly with respect to $n$. For this we first integrate by parts

$$
\begin{aligned}
& \left|\int_{X} k h \circ f^{n} d d^{c}\left(v_{\varepsilon}-v\right) \wedge T^{+}\right| \\
& \leq\left|\int_{X} d k \wedge d^{c}\left(v_{\varepsilon}-v\right) \wedge h \circ f^{n} T^{+}\right| \\
& \quad+\left|\int_{X} d h \circ f^{n} \wedge d^{c}\left(v_{\varepsilon}-v\right) \wedge k T^{+}\right|=I(n, \varepsilon)+I I(n, \varepsilon),
\end{aligned}
$$

and use the Cauchy-Schwarz inequality. For the first term we get

$$
I(n, \varepsilon) \leq\|h\|_{L^{\infty}}\|k\|_{e^{1}}\left[\int d\left(v_{\varepsilon}-v\right) \wedge d^{c}\left(v_{\varepsilon}-v\right) \wedge T^{+}\right]^{1 / 2} \rightarrow 0,
$$

because $v$ is bounded.

Treating the second integral $I I(n, \varepsilon)$ similarly gives rise to an integral of the form

$$
\int d\left(h \circ f^{n}\right) \wedge d^{c}\left(h \circ f^{n}\right) \wedge k T^{+} .
$$

Then we argue as in the proof of Theorem 3.3 in [DDG1] to get that this integral is $O\left(\lambda_{2}^{n} / \lambda_{1}^{n}\right)$.

Proposition 2.5. Let $h$ be a (smooth) test function on $X$. Then

$$
h \circ f^{n} T^{+} \rightarrow c T^{+}, \quad \text { where } c=\int h d \mu_{f}
$$

Again, the proof is similar to that of Theorem 3.3 in [DDG1], so we omit it.

2.3. Geometric intersection. In this paragraph, $X$ is supposed to be projective. We showed in [DDG1] that the invariant currents $T^{+}$and $T^{-}$admit important geometric structures. More precisely $T^{+}$(resp. $T^{-}$) is a strongly approximable laminar (resp. woven) current.

Our purpose here is to show that the measure $\mu_{f}$ can be alternatively obtained by "intersecting" these geometric structures. Recall that another result that has been obtained using laminarity is the fact that $T^{+}$-and consequently $\mu_{f}$-does not charge pluripolar sets. 
We first very briefly recall some preliminaries on geometric currents (see [Du1], [Du2] and also [DDG1], [DDG3] for more details). The construction of these geometric structures requires subdivisions $\mathcal{Q}$ of $X$ into families of "cubes" $Q \in \mathcal{Q}$, which are obtained by projecting along two generically transverse linear pencils, and taking intersections of subdivision of the projection bases into squares. In particular we have some freedom in the choice of the projections and the squares. The spaces parametrizing projections and squares are manifolds so we can speak of a "generic subdivision" where genericity is understood in the sense of Lebesgue measure.

That $T^{+}$is a strongly approximable laminar current means that if $\mathcal{Q}$ is a generic increasing family (actually, a sequence) of subdivisions by cubes, then $T^{+}$is the limit of an increasing family

$$
T_{\mathcal{Q}}^{+}=\sum_{Q \in \mathcal{Q}} T_{Q}^{+},
$$

the current $T_{Q}^{+}$is uniformly laminar in $Q$. Furthermore, $T_{Q}^{+}$can be written as an integral over a measured family of disjoint submanifolds of uniformly bounded volume

$$
T_{Q}^{+}=\int_{\alpha \in A_{Q}^{+}}\left[\Delta_{\alpha}^{+}\right] d v_{Q}^{+}(\alpha)
$$

We have the important estimate

$$
\mathbf{M}\left(T^{+}-T_{\mathcal{Q}}^{+}\right) \leq C r^{2} .
$$

The same holds for $T^{-}$, with "woven" instead of "laminar". In this case (8) becomes

$$
T_{Q}^{-}=\int_{\alpha \in A_{Q}^{-}}\left[\Delta_{\alpha}^{-}\right] d \nu_{Q}^{-}(\alpha),
$$

where the $\Delta^{-}$are allowed to intersect and can be singular. In view of (8) and (10) we can naturally define the geometric intersection of $T_{Q}^{+}$and $T_{Q}^{-}$as

$$
T_{Q}^{+} \dot{\wedge} T_{Q}^{-}=\int_{A_{Q}^{+}} \int_{A_{Q}^{-}}\left[\Delta_{\alpha}^{+} \cap \Delta_{\alpha^{\prime}}^{-}\right] d v_{Q}^{+}(\alpha) d v_{Q}^{-}\left(\alpha^{\prime}\right),
$$

where by definition, $\left[\Delta_{\alpha} \cap \Delta_{\alpha^{\prime}}\right]$ is the sum of Dirac masses at isolated intersections, counting multiplicities.

Assume now that $T^{+} \wedge T^{-}$is well defined, in the $L^{1}$ or energetic sense. We say that the wedge product $T^{+} \wedge T^{-}$is a geometric intersection if the family of measures $T_{\mathcal{Q}}^{+} \dot{\wedge} T_{\mathcal{Q}}^{-}:=\sum_{Q \in \mathcal{Q}} T_{Q}^{+} \dot{\wedge} T_{Q}^{-}$increases to $T^{+} \wedge T^{-}$when $\mathcal{Q}$ is any family of generic subdivisions into cubes of size $r \rightarrow 0$.

The following basic result asserts that for uniformly geometric currents the wedge product is geometric. 
Proposition 2.6. With notation as above, assume that $T_{\mathcal{Q}}^{+} \in L_{\mathrm{loc}}^{1}\left(T_{\mathcal{Q}}^{-}\right)$. Then the wedge product $T_{\mathbb{Q}}^{+} \wedge T_{\mathbb{Q}}^{-}$is geometric, that is,

$$
T_{\mathcal{Q}}^{+} \wedge T_{\mathcal{Q}}^{-}=\sum_{Q \in \mathcal{Q}} T_{Q}^{+} \dot{\wedge} T_{Q}^{-}
$$

Proof. The proposition follows by applying Lemma 2.7 below twice. Notice that if $\Delta$ and $\Delta^{\prime}$ are submanifolds in $Q,[\Delta] \in L_{\mathrm{loc}}^{1}\left(\left[\Delta^{\prime}\right]\right)$ iff $\Delta$ and $\Delta^{\prime}$ only have isolated intersections. If this holds, then $[\Delta] \wedge\left[\Delta^{\prime}\right]=\left[\Delta \cap \Delta^{\prime}\right]$ (see [De]).

Lemma 2.7. Assume that $R$ and $S$ are two positive closed currents in an open set $Q$, such that $S \in L_{\mathrm{loc}}^{1}(R)$ and $S$ admits a decomposition $S=\int S_{\alpha} d \nu(\alpha)$ as an integral of positive closed currents. Then for $v$ a.e. $\alpha, S_{\alpha} \in L_{\mathrm{loc}}^{1}(R)$ and we have the decomposition

$$
R \wedge S=\int\left(R \wedge S_{\alpha}\right) d v(\alpha)
$$

Proof. The result is local so consider a small ball $B \Subset Q$. By definition, $\alpha \mapsto$ $\mathbf{M}\left(\left.S_{\alpha}\right|_{B}\right)$ is locally $v$ integrable. By Lemma 2.8 below, for every $\alpha$ there exists a non positive psh function $u_{\alpha}$ in $B$ such that $d d^{c} u_{\alpha}=S_{\alpha}$ and $\left\|u_{\alpha}\right\|_{L^{1}\left(B^{\prime}\right)} \leq C \mathbf{M}\left(\left.S_{\alpha}\right|_{B}\right)$, where $B^{\prime}$ is a slightly smaller ball. Hence $u=\int u_{\alpha} d v(\alpha)$ is a well defined psh function which is a potential for $S$ in $B^{\prime}$.

Now since $u \in L_{\text {loc }}^{1}(R)$, we get that for a.e. $\alpha, u_{\alpha} \in L_{\text {loc }}^{1}(R)$, which is the first assertion of the lemma, while the second follows by applying $d d^{c}$ to the formula $u R=\int u_{\alpha} R d v(\alpha)$.

The following lemma is classical and goes back at least to [Le] (see [BE] for a brief treatment).

Lemma 2.8. Let $T$ be a positive closed current with finite mass in the unit ball $B \subset \mathbf{C}^{2}$. Then $T$ admits potential $u$ which is canonical, negative on $B(0,1 / 2)$ and satisfies $\|u\|_{L^{1}(B(0,3 / 4)} \leq C \mathbf{M}(T)$, with $C$ a universal constant.

We now arrive to the main result in this paragraph.

Theorem 2.9. Assume $X$ is projective and that $f$ has finite dynamical energy. Then the wedge product $T^{+} \wedge T^{-}$is geometric.

Proof. Let us first assume for simplicity that $T^{+} \in L^{1}\left(T^{-}\right)$. This guarantees that all the wedge products $T^{ \pm} \wedge T_{\mathcal{Q}}^{\mp}, T_{\mathcal{Q}}^{+} \wedge T_{\mathcal{Q}}^{-}$are well defined in the $L_{\text {loc }}^{1}$ sense and bounded from above by $T^{+} \wedge T^{-}$. The wedge product $T_{Q}^{+} \wedge T_{Q}^{-}$is geometric by Proposition 2.6 and we need to show that this product increases towards $\mu_{f}=T^{+} \wedge T^{-}$, as $r>0$ 
decreases towards 0 . Here the situation is symmetric so it suffices to estimate the mass $\mathbf{M}\left(T^{-} \wedge\left(T^{+}-T_{\mathcal{Q}}^{+}\right)\right)$. We follow the proof and notation of Theorem 5.2 in [Du2].

Shifting the cubes slightly, we can assume the mass of $\mu_{f}$ is not concentrated near the boundary of $\mathcal{Q}$. Let $\psi_{\mathcal{Q}}=\sum_{Q \in \mathcal{Q}} \psi_{Q}$ be a test function such that each function $\psi_{Q}$ is supported in the cube $Q$, satisfies $0 \leq \psi_{Q} \leq 1$ and is identically equal to 1 in the major part of $Q$. It suffices to show that $\int_{X} \psi_{Q} T^{-} \wedge\left(T^{+}-T_{Q}^{+}\right) \rightarrow 0$. Note that $\psi$ can be chosen so that $\left\|\nabla \psi_{\mathcal{Q}}\right\|=O(1 / r)$ and $\left\|d d^{c} \psi_{\mathcal{Q}}\right\|=O\left(1 / r^{2}\right)$.

Then

Let $G_{j}^{-}=\max \left\{G^{-},-j\right\}, T_{j}^{-}=\omega^{-}+d d^{c} G_{j}^{-}$and $\mu_{j}=T_{j}^{-} \wedge T^{+}$as before.

$$
\int \psi_{\mathcal{Q}} T^{-} \wedge\left(T^{+}-T_{\mathcal{Q}}^{+}\right)=\int \psi_{\mathcal{Q}} T_{j}^{-} \wedge\left(T^{+}-T_{\mathcal{Q}}^{+}\right)+\int \psi_{\mathcal{Q}}\left(T^{-}-T_{j}^{-}\right) \wedge\left(T^{+}-T_{\mathcal{Q}}^{+}\right) .
$$

In the second integral on the right, we use the facts that $T^{+} \geq T_{\mathcal{Q}}^{+}$are closed on Supp $\psi_{\mathcal{Q}}$ and that $T^{-} \wedge\left(T^{+}-T_{\mathcal{Q}}^{+}\right)=T_{j}^{-} \wedge\left(T^{+}-T_{\mathcal{Q}}^{+}\right)$on $\left\{G^{-}>-j\right\}$ to estimate

$$
\begin{aligned}
\left|\int_{X} \psi_{\mathcal{Q}}\left(T^{-}-T_{j}^{-}\right) \wedge\left(T^{+}-T_{\mathcal{Q}}^{+}\right)\right| & =\left|\int_{\left\{G^{-} \leq-j\right\}} \psi_{\mathcal{Q}}\left(T^{-}-T_{j}^{-}\right) \wedge\left(T^{+}-T_{\mathcal{Q}}^{+}\right)\right| \\
& \leq \int_{\left\{G^{-} \leq-j\right\}} \psi_{\mathcal{Q}}\left(\mu_{f}+\mu_{j}\right)
\end{aligned}
$$

which tends to zero as $j \rightarrow \infty$ uniformly in $r$ (recall that $\mu_{j}\left(\left\{G^{-} \leq-j\right\}\right)=$ $\mu\left(\left\{G^{-} \leq-j\right\}\right)$ see (5)). From now on we fix $j$ such that this integral is small.

It remains to control the first integral on the right hand side of (11). For convenience it is better to write $T_{j}^{-}=\theta^{-}+d d^{c} H_{j}$, where $\theta^{-}$is a smooth form cohomologous to $\omega^{-}$and $H_{j}$ is still bounded. This gives

$$
\int \psi_{\mathcal{Q}} T_{j}^{-} \wedge\left(T^{+}-T_{\mathcal{Q}}^{+}\right)=-\int d \psi_{\mathcal{Q}} \wedge d^{c} H_{j} \wedge\left(T^{+}-T_{\mathcal{Q}}^{+}\right)+\int \psi_{\mathcal{Q}} \theta^{-} \wedge\left(T^{+}-T_{\mathcal{Q}}^{+}\right) .
$$

The second term on the right tends to zero as $r \rightarrow 0$ by laminarity of $T^{+}$. The first we estimate with Schwarz' inequality and the estimate given by the "strong approximability" of $T^{+}$.

$$
\left|\int d \psi_{\mathcal{Q}} \wedge d^{c} H_{j} \wedge\left(T^{+}-T_{\mathcal{Q}}^{+}\right)\right|^{2} \leq\left\|d \psi_{\mathcal{Q}}\right\|_{\infty}^{2} O\left(r^{2}\right) \int d H_{j} \wedge d^{c} H_{j} \wedge\left(T^{+}-T_{\mathcal{Q}}^{+}\right) .
$$

Since $\left\|d \psi_{Q}\right\|_{\infty}^{2}=O\left(r^{-2}\right)$, it suffices to know that the last integral tends to zero with $r$. But this happens because the bounded function $H_{j}$ has gradient in $L^{2}$ with respect to $T^{+}$, and because $T_{\mathcal{Q}}^{+} \nearrow T^{+}$(see the end of the argument in [Du2]). 
We now no longer assume that $T^{+} \in L^{1}\left(T^{-}\right)$. One difficulty is that we have to justify the existence of the local wedge products $T_{Q}^{+} \wedge T^{-}$, etc. This is ensured by the following lemma (which, it is perhaps worth stressing, does not require homogeneity of $X$ ).

Lemma 2.10. There exists $S_{Q}^{+}$arbitrary close to $T_{Q}^{+}$such that $G^{-} \in L_{\mathrm{loc}}^{1}\left(S_{Q}^{+}\right)$. Likewise, there exists $S_{\mathscr{Q}}^{-}$arbitrary close to $T_{\mathcal{Q}}^{-}$such that $G^{+} \in L_{\mathrm{loc}}^{1}\left(S_{\mathcal{Q}}^{-}\right)$.

Proof. Since $T^{-} \in \mathcal{E}\left(T^{+}\right)$, by the first item of Proposition $1.2 G^{-}$is not $-\infty$ a.e. on $T^{+}$. Thus, given a cube $Q, G^{-}$is not identically $-\infty$ on $T_{Q}^{+}$. Now $T_{Q}^{+}=$ $\int\left[\Delta_{\alpha}\right] d v_{Q}^{+}(\alpha)$, hence for $v^{+}$-a.e. disk $\Delta_{\alpha}, \Phi(\alpha):=\int_{\Delta_{\alpha}} G^{-}$is finite. Consider now

$$
v_{N}^{+}:=\left.v^{+}\right|_{\{\Phi>-N\}} \text { and } T_{Q, N}^{+}:=\int\left[\Delta_{\alpha}\right] d v_{N}^{+}(\alpha) .
$$

Then by construction $G^{-} \in L^{1}\left(T_{Q, N}^{+}\right)$and $T_{Q, N}^{+}$is arbitrarily close to $T_{Q}^{+}$.

The argument for $S_{\mathbb{Q}}^{-}$is similar.

From now on we replace the $T_{\mathcal{Q}}^{ \pm}$with the $S_{\mathcal{Q}}^{ \pm}$as given by the previous lemma, so all the local wedge products $\left(T_{Q}^{+} \wedge T_{Q}^{-}, T_{Q}^{+} \wedge T^{-}, T^{+} \wedge T_{Q}^{-}\right)$are well defined in the usual $L^{1}$ sense. We further assume that $S_{Q}^{ \pm}$is so close to $T_{Q}^{ \pm}$that the estimate (9) is satisfied.

We now need to justify the inequalities $T_{\mathcal{Q}}^{+} \wedge T_{\mathcal{Q}}^{-} \leq T^{+} \wedge T^{-}$, etc. This follows from the following simple observation: let $T_{j}^{-}=\omega^{-}+d d^{c} \max \left(G^{-},-j\right)$. We have that $T^{+} \wedge T_{j}^{-} \rightarrow T^{+} \wedge T^{-}$(by the energy approach) and $T_{\mathcal{Q}}^{+} \wedge T_{j}^{-} \rightarrow T_{\mathcal{Q}}^{+} \wedge T^{-}$(by the classical approach) as $j \rightarrow \infty$. Since $T_{Q}^{+} \wedge T_{j}^{-} \leq T^{+} \wedge T_{j}^{-}$we conclude that $T_{Q}^{+} \wedge T^{-} \leq T^{+} \wedge T^{-}$. In the same way we obtain that $T^{+} \wedge T_{\mathcal{Q}}^{-} \leq T^{+} \wedge T^{-}$. The inequality $T_{\mathcal{Q}}^{+} \wedge T_{\mathcal{Q}}^{-} \leq T_{Q}^{+} \wedge T^{-}$is obvious since all wedge products are defined in the classical sense.

Starting from here the proof is identical to the $L^{1}$ case.

\section{Homogeneous weights}

In this section we give criteria allowing to verify in practice (see $\$ 4.4$ ) the finite energy condition for the homogeneous weights $\chi(t)=-(-t)^{p}$, with $0<p \leq 1$.

Recall from (6) and (7) that we can write $T^{ \pm}=\omega^{ \pm}+d d^{c} G^{ \pm}$. To analyze the potential $G^{+}$, it is easier to use the function $\Gamma^{+}$as an intermediary. Indeed, Proposition 2.4 in [DDG1] implies that $\Gamma^{+} \geq A \log \operatorname{dist}\left(\cdot, I^{+}\right)-B$ for some $A, B>$ 0 , where $I^{+} \subset X$ is the indeterminacy set of the map $f$. Let us call an indeterminacy point

$$
p \in I^{+} \text {spurious if } f(p) \cdot \omega^{+}=0 .
$$


At every non-spurious point, we also have the reverse inequality

$$
\Gamma^{+} \leq A \log \operatorname{dist}(\cdot, p)-B
$$

Similar inequalities hold for $\Gamma^{-}$. We let $E^{+}$denote the exceptional set of $f$, i.e. the union of those curves collapsed by $f$ to points, and set $I^{-}=f\left(E^{+}\right)$. Then $\Gamma^{-} \geq A \log \operatorname{dist}\left(\cdot, I^{-}\right)-B$ with the reverse inequality holding if and only $f^{-1}(p) \cdot \omega^{-} \neq 0$ for every $p \in I^{-}$. Likewise, we say that a point

$$
p \in I^{-} \text {is spurious if } f^{-1}(p) \cdot \omega^{-}=0 .
$$

We refer the reader to [DDG1] for more about spurious points. Here we point out only that

(1) if the invariant cohomology classes $\left\{T^{+}\right\},\left\{T^{-}\right\}$are Kähler, then there is no spurious point of indeterminacy in resp. $I^{+}, I^{-}$;

(2) when $f$ is bimeromorphic, we can always perform a bimeromorphic change of coordinates to get rid of spurious indeterminacy ([BD], Proposition 4.1).

It is plausible that a similar result holds when the topological degree $\lambda_{2}(f)$ is merely smaller than the first dynamical degree $\lambda_{1}=\lambda_{1}(f)$. We refer the reader to $\S 5$ in [DDG1] for some results in this direction.

The Lelong numbers of $T^{+}$vanish on $I^{-}$(see [DDG1], Theorem 2.4). Hence Proposition 1.20 gives

Proposition 3.1. We have $\Gamma^{-} \in \mathcal{E}\left(T^{+}, \omega^{-}\right)$.

A similar result holds for $\Gamma^{+} / T^{-}$if we know that $T^{-}$has zero Lelong number at each point in $I^{+}$(which we do e.g. when $\lambda_{2}(f)=1$ ).

The main theme in this section is that for homogeneous weights, it is possible to pass from control on energy of $\Gamma^{ \pm}$to control on that of $G^{ \pm}$. This idea originates in [BD]. We set

$$
\mathcal{E}^{p}(T, \omega):=\mathcal{E}_{\chi}(T, \omega) \text { and } \nabla^{p}(T, \omega):=\nabla_{\chi}(T, \omega)
$$

where $\chi(t)=-(-t)^{p}, 0<p \leq 1$.

Proposition 3.2. Fix $0<p \leq 1$. Then

$$
\Gamma^{+} \in \mathcal{E}^{p}\left(T^{-}, \omega^{+}\right) \text {if and only if } G^{+} \in \nabla^{p}\left(T^{-}, \omega^{+}\right) .
$$

If $\lambda_{1}^{p}>\lambda_{2}(f)$ then we similarly have that

$$
\Gamma^{-} \in \mathcal{E}^{p}\left(T^{+}, \omega^{-}\right) \text {if and only if } G^{-} \in \nabla^{p}\left(T^{+}, \omega^{-}\right) .
$$


Proof. Since $\Gamma^{+}$and $G^{+}$are both $\omega^{+}$-psh functions such that $G^{+} \leq \Gamma^{+} \leq 0$, it follows from Corollary 1.15 that

$$
G^{+} \in \mathcal{E}_{\chi}\left(T^{-}, \omega^{+}\right) \Longrightarrow \Gamma^{+} \in \mathcal{E}_{\chi}\left(T^{-}, \omega^{+}\right),
$$

for any weight $\chi \in W$.

Assume conversely now that $\Gamma^{+} \in \mathcal{E}_{\chi}\left(T^{-}, \omega^{+}\right)$for the special homogeneous weight $\chi(t)=-(-t)^{p}, 0<p \leq 1$. Since $\chi^{\prime} \circ G^{+} \leq \chi^{\prime}\left(\lambda_{1}^{-j} \Gamma^{+} \circ f^{j}\right)=$ $\lambda_{1}^{-j(p-1)} \chi^{\prime}\left(\Gamma^{+} \circ f^{j}\right)$, it follows from the Cauchy-Schwarz inequality and $\left(f^{j}\right)_{*} T^{-}=\lambda_{1}^{j} T^{-}$that

$$
\begin{aligned}
& \left(\int_{X} \chi^{\prime} \circ G^{+} d G^{+} \wedge d^{c} G^{+} \wedge T^{-}\right)^{1 / 2} \\
& \quad \leq \sum_{j \geq 0} \frac{1}{\lambda_{1}^{j}}\left(\int_{X} \chi^{\prime} \circ G^{+} d\left(\Gamma^{+} \circ f^{j}\right) \wedge d^{c}\left(\Gamma^{+} \circ f^{j}\right) \wedge T^{-}\right)^{1 / 2} \\
& \quad \leq \sum_{j \geq 0} \frac{1}{\lambda_{1}^{j p / 2}}\left(\int_{X} \chi^{\prime} \circ \Gamma^{+} d \Gamma^{+} \wedge d^{c} \Gamma^{+} \wedge T^{-}\right)^{1 / 2}<+\infty .
\end{aligned}
$$

Hence $G^{+} \in \nabla_{\chi}\left(T^{-}, \omega^{+}\right)$.

For $\Gamma^{-}$and $G^{-}$, the proof is very similar except that in the estimate analogous to the one in the previous display, pushforward does not distribute over products as well as pullback. If $\eta$ is a $(1,0)$ form, we have only an inequality

$$
i f_{*} \eta \wedge \overline{f_{*} \eta} \leq \lambda_{2}(f) \cdot f_{*}(i \eta \wedge \bar{\eta}) \text {. }
$$

Arguing as above and setting $E_{\chi}\left(\Gamma^{-}\right)=\int \chi^{\prime} \circ \Gamma^{-} d \Gamma^{-} \wedge d^{c} \Gamma^{-} \wedge T^{+}$, we thus get

$$
\left(\int_{X} \chi^{\prime} \circ G^{-} d G^{-} \wedge d^{c} G^{-} \wedge T^{+}\right)^{1 / 2} \leq \sum_{j \geq 0}\left(\frac{\lambda_{2}}{\lambda_{1}^{p}}\right)^{j / 2}\left[E_{\chi}\left(\Gamma^{-}\right)\right]^{1 / 2},
$$

which is finite if $\lambda_{1}^{p}>\lambda_{2}$.

Since $L^{1} \subset L^{p}$ for $0<p \leq 1$, Propositions 1.17 and 3.2 directly imply

Corollary 3.3. Suppose $0<p \leq 1$ and $T^{+} \in L^{1}\left(T^{-}\right)$. Then $\Gamma^{+}$belongs to $\mathscr{E}^{p}\left(T^{-}, \omega^{+}\right)$if and only if $G^{+}$does. If in addition $\lambda_{1}^{p}>\lambda_{2}$, then $\Gamma^{-}$belongs to $\varepsilon^{p}\left(T^{+}, \omega^{-}\right)$if and only if $G^{-}$does.

Having largely reduced the problem of controlling $G^{ \pm}$to that of controlling $\Gamma^{ \pm}$, we now seek effective means of accomplishing the latter. The computations below will be the same for $\Gamma^{+}$and $\Gamma^{-}$, so we work only with $\Gamma^{+}$. We let $\Omega=\omega^{+}+c \omega$. For $c>0$ large enough, both $\Gamma^{+}$and $\varphi:=\log \operatorname{dist}\left(\cdot, I^{+}\right)$are $\Omega$-psh functions. Since $\Gamma^{+} \geq A \varphi-B$, Lemma 1.13 gives 
Proposition 3.4. Let $\chi$ be any weight function. Then $\varphi \in \mathcal{E}_{\chi}\left(T^{-}, \Omega\right)$ implies that $\Gamma^{+} \in \mathcal{E}_{\chi}\left(T^{-}, \Omega\right)$.

Hence we consider weighted energy of $\varphi$. Let $\varphi_{j}$ be the canonical approximants. Then up to finite additive constants we have

$$
\begin{aligned}
0 \leq & -\int \chi \circ \varphi_{j}\left(\Omega+d d^{c} \varphi_{j}\right) \wedge T^{-}=-\int-G^{-} d d^{c} \varphi_{j} \wedge d d^{c} \chi \circ \varphi_{j} \\
= & \int-G^{-}\left(\chi^{\prime \prime} \circ \varphi_{j}\right) d \varphi_{j} \wedge d^{c} \varphi_{j} \wedge d d^{c} \varphi_{j}+\int-G^{-}\left(\chi^{\prime} \circ \varphi_{j}\right)\left(d d^{c} \varphi_{j}\right)^{2} \\
\leq & \int_{X \backslash I^{+}}-G^{-} \chi^{\prime \prime} \circ \varphi d \varphi \wedge d^{c} \varphi \wedge d d^{c} \varphi \\
& \quad+\int_{X \backslash I^{+}}-G^{-} \chi^{\prime} \circ \varphi\left(d d^{c} \varphi\right)^{2}+\int-G^{-}\left(\chi^{\prime} \circ \varphi\right) v_{j} \\
= & \mathrm{I}+\mathrm{II}+\mathrm{III},
\end{aligned}
$$

where $v_{j}=\left.\left(d d^{c} \varphi_{j}\right)^{2}\right|_{\varphi=-j}$ is a positive measure. Note also that $d \varphi_{\wedge} d^{c} \varphi_{j} \wedge d d^{c} \varphi_{j}^{2}$ puts no mass on $\varphi=-j$. One can verify this by replacing $\max \{\cdot, j\}$ with a smooth convex approximation in the definition of $\varphi_{j}$ and then computing directly.

To bound integral $\mathrm{I}$, we compute in local coordinates that

$$
d \varphi \wedge d^{c} \varphi \wedge d d^{c} \varphi \leq \frac{C d V(x)}{\operatorname{dist}\left(x, I^{+}\right)^{4}}
$$

So if $m^{-}(t)$ is the spherical mean of $G^{-}$on the set $\left\{\operatorname{dist}\left(p, I^{+}\right)=e^{t}\right\}$, we get

$$
I \leq C \int_{-\infty}^{\max \varphi}-m^{-}(t) \chi^{\prime \prime}(t) d t,
$$

We claim that II is always finite. To see this, let $\pi: \hat{X} \rightarrow X$ be the blowup of $X$ along the finite set $I^{+}$. Then direct computation in local coordinates about $I^{+}$reveals that on $X \backslash I^{+}$, one has $\left(d d^{c} \varphi \circ \pi\right)^{2} \leq d V$, where $d V$ is a smooth volume form on $\hat{X}$. Since $G^{-} \circ \pi$ is $\pi^{*} \alpha^{-}$-psh on $\hat{X}$, this gives us that

$$
\mathrm{II}=\int_{\hat{X} \backslash \pi^{-1}\left(I^{+}\right)}-\left(G^{-} \circ \pi\right)\left(\chi^{\prime} \circ \varphi \circ \pi\right)\left(d d^{c} \varphi \circ \pi\right)^{2} \leq \int_{\hat{X} \backslash \pi^{-1}\left(I^{+}\right)}-G^{-} \circ \pi d V<\infty .
$$

Finally, to deal with III, we note that for $j$ large $v_{j}$ is uniformly (in $j$ ) proportional to normalized spherical measure on $\left\{\operatorname{dist}\left(p, I^{+}\right)=e^{-j}\right\}$. Hence we obtain that

$$
\mathrm{III} \leq-C m^{-}(-j) \chi^{\prime}(-j) .
$$

With these estimates we arrive at two conclusions very much in the spirit of [BD]. We state them only for $\Gamma^{+}$, but the analogous assertions for $\Gamma^{-}$are equally valid and proved in the same way. 
Corollary 3.5. If $G^{-}$is finite at all points in $I^{+}$, then $\Gamma^{+} \in \mathcal{E}^{1}\left(T^{-}, \omega^{+}\right)$. Hence also $G^{+} \in \nabla^{1}\left(T^{-}, \omega^{+}\right)$. If moreover $T^{+} \in L^{1}\left(T^{-}\right)$then $G^{+} \in \mathcal{E}^{1}\left(T^{-}, \omega^{+}\right)$.

Proof. By Propositions 3.4 and 3.2, it suffices to verify that the above bounds on I and III are finite (and uniform as $j \rightarrow \infty$ ). In this case, we have $\chi(t)=t$, and in particular $\chi^{\prime \prime} \equiv 0$. Hence I is trivially finite.

On the other hand, $\chi^{\prime} \equiv 1$ and as $t \rightarrow-\infty, m^{-}(t)$ decreases to the average value of $G^{-}$on the set $I^{+}$. In particular the bound on III is uniform as $j \rightarrow \infty$ if $G^{-}$is finite on $I^{+}$.

For other homogeneous weight functions, our estimates on I and III immediately give an analogous criterion.

Corollary 3.6. Let (as above) $m^{-}(t)$ denote the mean of $G^{-}$on $\left\{\operatorname{dist}\left(x, I^{+}\right)=e^{t}\right\}$. Suppose for some $q \in(0,1)$ that

$$
\limsup _{t \rightarrow-\infty}|t|^{q-1}\left|m^{-}(t)\right|<\infty .
$$

Then for any $0<p<q$, we have $\Gamma^{+} \in \mathcal{E}^{p}\left(T^{-}, \omega^{+}\right)$. Hence $G^{+} \in \nabla^{p}\left(T^{-}, \omega^{+}\right)$. If additionally $T^{+} \in L^{1}\left(T^{-}\right)$, then $G^{+} \in \mathcal{E}^{p}\left(T^{-}, \omega^{+}\right)$.

In some circumstances (e.g. polynomial maps of $\mathbb{C}^{2}$ ) it is possible to see directly that $T^{+} \in L^{1}\left(T^{-}\right)$. Corollaries 3.5 and 3.6 are adequate by themselves for these situations. In other circumstances, however, it is not easy to verify that $T^{+} \in L^{1}\left(T^{-}\right)$. We show now that one can avoid doing this if there are no spurious points in $I^{+}$or $I^{-}$.

Theorem 3.7. Suppose that $0<p \leq 1$ is chosen so that $\lambda_{1}^{p}>\lambda_{2}$ and that $G^{+} \in$ $\nabla^{p}\left(T^{-}, \omega^{+}\right), G^{-} \in \nabla^{p}\left(T^{+}, \omega^{-}\right)$. If there are no spurious points in $I^{+}$or $I^{-}$, then it is further true that $G^{+} \in \mathcal{E}^{p}\left(T^{-}, \omega\right)$ and $G^{-} \in \mathcal{E}^{p}\left(T^{+}, \omega\right)$.

We prove the theorem in a sequence of lemmas, focusing mainly on $G^{+}$. The case of $G^{-}$is identical, except that as in the proof of Proposition 3.2, we need the condition $\lambda_{1}^{p}>\lambda_{2}$ to make the triangle inequality work in a couple of places below.

Lemma 3.8. There exists $k \in \mathbb{N}$ and $c>0$ such that for every $n \in \mathbb{N}$, we have

$$
\frac{f_{*}^{n k} \omega}{\lambda_{1}^{n k}} \leq c \omega+d d^{c} w_{n}
$$

where $w_{n} \geq-c G^{-}$is $c \omega$-psh

We remark before continuing that if the indeterminacy set of $f$ has no spurious points, then neither does that of $f^{k}$. Therefore, there is no particular harm in assuming for the sake of notational simplicity that $k=1$ when we apply the lemma. 
Proof. Let $\theta^{-}$be a smooth form cohomologous to $T^{-}$. Then by Theorem 1.3 in [DDG1] we have $\omega=a \theta^{-}+\eta$ for some $a>0$ and a smooth form $\eta$ such that $\lambda_{1}^{-k} f_{*}^{k} \eta$ tends to zero in cohomology. Thus for $k$ large enough, we have

$$
\frac{f_{*}^{k} \omega}{\lambda_{1}^{k}} \leq a \theta^{-}+\frac{1}{2} \omega+d d^{c} w
$$

where $w$ is a quasipsh function that is smooth away from $I^{-}\left(f^{k}\right)$. Since there are no spurious points in $I^{-}$, we have in fact that $w \geq b G^{-}$for some $b>0$. Iterating this inequality then gives

$$
\begin{array}{r}
\frac{f_{*}^{k n} \omega}{\lambda_{1}^{k n}} \leq a b_{n} \theta^{-}+\frac{1}{2^{n}} \omega+d d^{c}\left(\sum_{j=0}^{n-1} \frac{f_{*}^{j k} w}{2^{(n-1-j)} \lambda_{1}^{j k}}\right) \\
+d d^{c}\left(\sum_{j=0}^{n-2} a b_{n-2-j} \frac{1}{\lambda_{1}^{j k}}\left(f^{j k}\right)_{*} \Gamma^{-}\right),
\end{array}
$$

where $b_{n}=\sum_{\ell=0}^{n-1} 2^{-\ell} \leq 2$. Since $\frac{f_{*}^{j} \Gamma^{-}}{\lambda_{1}^{j}} \geq \frac{f_{*}^{j} G^{-}}{\lambda_{1}^{j}} \geq G^{-}$, the lemma follows immediately.

Lemma 3.9. Both $\int-\chi \circ G^{+} T^{+} \wedge \omega$ and $\int-\chi \circ G^{-} T^{-} \wedge \omega$ are finite.

Proof. Modulo finite additive constants, the first integral is estimated by

$$
\begin{aligned}
\left(\int\right. & \left.-\chi \circ G^{+} T^{+} \wedge \omega\right)^{1 / 2} \\
& =\left(\int \chi^{\prime} \circ G^{+} d G^{+} \wedge d^{c} G^{+} \wedge \omega\right)^{1 / 2} \\
& \leq \sum_{n=1}^{\infty}\left(\int \chi^{\prime}\left(\frac{\Gamma^{+} \circ f^{n}}{\lambda_{1}^{n}}\right) \frac{d\left(\Gamma^{+} \circ f^{n}\right) \wedge d^{c}\left(\Gamma^{+} \circ f^{n}\right)}{\lambda_{1}^{2 n}} \wedge \omega\right)^{1 / 2} \\
& =\sum_{n=1}^{\infty} \frac{1}{\lambda_{1}^{n p / 2}}\left(\int \chi^{\prime} \circ \Gamma^{+} d \Gamma^{+} \wedge d^{c} \Gamma^{+} \wedge \frac{f_{*}^{n} \omega}{\lambda_{1}^{n}}\right)^{1 / 2} \\
& \leq \sum_{n=1}^{\infty} \frac{1}{\lambda_{1}^{n p / 2}}\left(\int \chi^{\prime} \circ \Gamma^{+} d \Gamma^{+} \wedge d^{c} \Gamma^{+} \wedge\left(c \omega+d d^{c} w_{n}\right)\right)^{1 / 2}
\end{aligned}
$$

where $c, w_{n}$ are as in the previous lemma. If we take the contributions to the integral from $c \omega$ and from $d d^{c} w_{n}$ separately, then the first contribution is finite by 
Proposition 1.20. The second contribution is handled up to additive constants as follows:

$$
\begin{aligned}
\int \chi^{\prime} & \circ \Gamma^{+} d \Gamma^{+} \wedge d^{c} \Gamma^{+} \wedge d d^{c} w_{n} \\
& =\int-w_{n} d d^{c}\left(\chi \circ \Gamma^{+}\right) \wedge d d^{c} \Gamma^{+} \\
& =\int-w_{n}\left(d d^{c} \chi \circ \Gamma^{+}+\omega^{+}\right) \wedge\left(d d^{c} \Gamma^{+}+\omega^{+}\right)+O(1) \\
& \leq c \int-G^{-}\left(d d^{c} \chi \circ \Gamma^{+}+\omega^{+}\right) \wedge\left(d d^{c} \Gamma^{+}+\omega^{+}\right)+O(1) \\
& =c \int-G^{-} d d^{c}\left(\chi \circ \Gamma^{+}\right) \wedge d d^{c} \Gamma^{+}+O(1) \\
& =c \int \chi^{\prime} \circ \Gamma^{+} d \Gamma^{+} \wedge d^{c} \Gamma^{+} \wedge T^{-}+O(1)
\end{aligned}
$$

which is finite by the hypotheses of Theorem 3.7

Lemma 3.10. Both $\int-\chi \circ G^{+} T^{-} \wedge \omega$ and $\int-\chi \circ G^{-} T^{+} \wedge \omega$ are finite.

Proof. We treat the first integral only. Let $\Omega=\omega^{+}+\omega^{-}$. Then from Proposition 2.5 in [GZ2] we have up to additive constants that

$$
\begin{aligned}
\int-\chi \circ G^{+} T^{-} \wedge \omega= & \int-\chi \circ G^{+} d d^{c}\left(G^{-}+\Omega\right) \wedge \omega \\
\leq & 2 \int-\chi \circ G^{+}\left[\Omega+d d^{c} G^{+}\right] \wedge \omega \\
& \quad+2 \int-\chi \circ G^{-}\left[\Omega+d d^{c} G^{-}\right] \wedge \omega . \\
= & 2 \int-\chi \circ G^{+} T^{+} \wedge \omega+2 \int-\chi \circ G^{-} T^{-} \wedge \omega .
\end{aligned}
$$

We have just seen that the last two integrals are finite, so the proof is complete.

Theorem 3.7 is now an immediate consequence of Proposition 1.17 and Remark 1.18 .

\section{Examples}

In this section we exhibit families of examples satisfying our energy conditions. Recall from Theorem 4.2 in [DDG1] that the assumption $\lambda_{1}(f)>\lambda_{2}(f)$ implies that $X$ is either rational or of Kodaira dimension zero. 
4.1. Polynomial mappings of $\mathbf{C}^{2}$. Suppose that $f: \mathbb{C}^{2} \rightarrow \mathbb{C}^{2}$ is polynomial and, as always, has small topological degree. Recent work of Favre and Jonsson [FJ] gives a smooth compactification $X=\mathbb{C}^{2} \cup D_{\infty}$ of $\mathbb{C}^{2}$ and $k \in \mathbb{N}^{*}$ such that

(P1) the meromorphic extension of $f^{k}$ to $X$ is 1-stable;

(P2) $f^{k}$ contracts the divisor $D_{\infty}$ at infinity to a fixed point in $I^{-} \backslash I^{+}\left(f^{k}\right)$;

(P3) the relative potential $G^{+}$for $T^{+}$is continuous in $X \backslash I^{+}\left(f^{k}\right)$.

Using this information, we will show

Theorem 4.1. Let $f: \mathbb{C}^{2} \rightarrow \mathbb{C}^{2}$ be a polynomial mapping with $\lambda_{2}(f)<\lambda_{1}(f)$, and let $X, k$ be as in (P1)-(P3). Then $f^{k}: X \rightarrow X$ has finite dynamical energy. More precisely $G^{+} \in \mathcal{E}^{1}\left(T^{-}, \omega^{+}\right)$and $G^{-} \in \mathcal{E}^{1}\left(T^{+}, \omega^{-}\right)$.

Proof. To simplify notation, we replace $f^{k}$ by $f$. We recall that 1 -stability implies that $I^{+} \cap I^{-}=\emptyset$. Since $G^{+}$is continuous in $X \backslash I^{+}$, it is in particular finite at all points in $I^{-}$, so $G^{+} \in \mathcal{E}^{1}\left(T^{-}, \omega^{+}\right)$by Proposition 1.20 and Corollary 3.5.

Though $G^{-}$is less well-behaved, we can show it is finite at all points in $I^{+}$. Since $f$ is polynomial on $\mathbb{C}^{2}$, we have $I^{+} \subset D_{\infty}$. The invariance $f\left(\mathbb{C}^{2}\right) \subset \mathbb{C}^{2}$ and the contraction property $f\left(D_{\infty} \backslash I^{+}\right)=q=f(q) \in I^{-}$imply that $f^{-1}\left(I^{+}\right) \subset I^{+}$. Thus

$$
G^{-}(p)=\sum_{n \geq 0} \frac{1}{\lambda_{1}^{n}}\left(f^{n}\right)_{*} \Gamma^{-}(p) \geq \sum_{n \geq 0} \frac{1}{\lambda_{1}^{n}}\left(f^{n}\right)_{*} M>-\infty,
$$

where $M=\min _{p \in I^{+}} \Gamma^{-}(p)$ is finite because $I^{+} \cap I^{-}=\emptyset$ (see Lemma 3.2 in [DDG1]). From Corollary 3.5 again, we see that $G^{-} \in \nabla^{1}\left(T^{+}, \omega^{-}\right)$. Since $G^{+} \in$ $L^{1}\left(T^{-}\right)$, we have $G^{-} \in L^{1}\left(T^{+}\right)$by symmetry. Hence in fact $G^{-} \in \mathcal{E}^{1}\left(T^{+}, \omega^{-}\right)$.

It follows from Theorem 2.4 that $\mu_{f}=T^{+} \wedge T^{-}$is a well-defined, mixing invariant probability measure which does not charge pluripolar sets. We can actually say more.

Theorem 4.2. Let $f$ be as in Theorem 4.1, and $V \subset X$ be an algebraic curve. Then $\log \operatorname{dist}(\cdot, V) \in L^{1}\left(\mu_{f}\right)$.

In particular $\log \operatorname{dist}\left(\cdot, \boldsymbol{C}_{f}\right) \in L^{1}\left(\mu_{f}\right)$, where $\boldsymbol{C}_{f}$ denotes the critical set of $f$. This result will allow us in [DDG3] to use Pesin's theory of non-uniformly hyperbolic dynamical systems and show the existence of many saddle periodic points.

Proof. Assume without loss of generality that $V$ is irreducible. We claim that $G_{\mid V}^{-} \not \equiv$ $-\infty$ on $V$. Granting this for the moment, let $0 \geq \varphi_{V} \in L^{1}(X)$ be a global potential for the current of integration along $V$. Thus $d d^{c} \varphi_{V}=[V]-\Theta$, where $\Theta$ is smooth, 
and $\varphi_{V} \in L^{1}\left(\mu_{f}\right)$ if and only if $\log \operatorname{dist}(\cdot, V) \in L^{1}\left(\mu_{f}\right)$. From our claim we have that $G^{-} \in L^{1}([V])$ and, by symmetry $\varphi \in L^{1}\left(T^{-}\right)$. Hence the measure $T^{-} \wedge[V]$ is well-defined.

Fix a constant $\gamma$ between $\sqrt{\lambda_{2}}$ and $\lambda_{1}$. Then from [FJ], we have $C_{1}>0$ such that

$$
\operatorname{dist}\left(f^{n} x, I^{+}\right) \geq\left(C_{1} \operatorname{dist}\left(x, I^{+}\right)\right)^{\gamma^{n}}, \quad \text { for all } x \in X \text { and } n \in \mathbb{N}
$$

Since $\Gamma^{+}(x) \geq A \log \operatorname{dist}\left(x, I^{+}\right)-B$, we infer from (6) that

$$
G^{+}(x) \geq A^{\prime} \log \operatorname{dist}\left(x, I^{+}\right)-B^{\prime} .
$$

Therefore repeated integration by parts gives (up to finite additive/multiplicative constants)

$$
\begin{aligned}
\int-\varphi \mu_{f} & =\int-G^{+} T^{-} \wedge[V] \leq \int-\log \operatorname{dist}\left(\cdot, I^{+}\right) T^{-} \wedge[V] \\
& =\int_{V}-G^{-} d d^{c} \log \operatorname{dist}\left(\cdot, I^{+}\right) \wedge[V] \\
& \leq \int_{V}-G^{-} \omega \wedge[V]+\sum_{x \in I^{+} \cap V} G^{-}(x)<\infty,
\end{aligned}
$$

since $G^{-}$is finite on $I^{+}$.

It remains to verify that $G^{-}$is not identically $-\infty$ on $V$. Since $V$ must meet $D_{\infty}$ somewhere, it suffices to show that $G^{-}$is finite on $V \cap D_{\infty}$. This is the case if, for instance, $V \cap D_{\infty} \subset I^{+}$. However, from $f_{*} G^{-}=\lambda_{1}\left(G^{-}-\Gamma^{-}\right)$, we see that we need only show that $G^{-}$is finite on $f^{-n}(V) \cap D_{\infty}$ for some $n \in \mathbb{N}$. If, for instance, $V \cap D_{\infty}$ does not contain the superattracting point $q \in I^{-}$, then this is true for $n=1$ because $f^{-1}\left(D_{\infty} \backslash\{q\}\right) \subset I^{+}$. Finally, as the next lemma makes clear, even if $D_{\infty} \cap V$ does contain $q$, the same reasoning works for some larger value of $n$.

Lemma 4.3. There exists an integer $n \in \mathbb{N}$ such that if $W$ is any irreducible component of $f^{-n}(V)$, not contained in $D_{\infty}$, then $q \notin W$.

Proof. Suppose on the contrary that for each $n \in \mathbb{N}$, there exists an irreducible component $W_{n}$ of $f^{n *} V$ such that $q \in W_{n}$ but $W_{n} \not \subset D$. Then since $q$ is superattracting for $f$, this remains true at the local level. That is, there is a neighborhood $U \ni q$ and for every $n \in \mathbb{N}$ a local irreducible component $W_{n}$ of the (local) pullback $\left(\left.f\right|_{U}\right)^{n *}(V)$ such that $q \in W_{n}$ but $W_{n} \not \subset D$.

Moreover, by [FJ] we may choose a coordinate patch $U$ about $q=\mathbf{0}$ so that $f:(U, \mathbf{0}) \rightarrow(U, \mathbf{0})$ is a rigid holomorphic germ and there exists a non-trivial local 
divisor $D_{\text {loc }} \subset D$ satisfying $\left(\left.f\right|_{U}\right)^{*} D_{\text {loc }} \geq \lambda_{1} D_{\text {loc }}$. The local topological degree of $f_{\mid U}$ is no larger than $\lambda_{2}$. Hence

$$
\lambda_{1}^{n} \leq\left\langle W_{n}, f^{n *} D_{\mathrm{loc}}\right\rangle_{q}=\left\langle f_{*}^{n} W_{n}, D_{\mathrm{loc}}\right\rangle_{q} \leq \lambda_{2}^{n}\left\langle V, D_{\mathrm{loc}}\right\rangle_{q},
$$

where $\langle\cdot, \cdot\rangle_{q}$ denotes local intersection of germs at $q$. This contradicts $\lambda_{2}<\lambda_{1}$ for large $n$.

4.2. The secant method. If $P: \mathbb{C} \rightarrow \mathbb{C}$ is a polynomial of degree at least two such that all roots of $P$ are simple, then we recall the secant method from [DDG1]: given points $x, y \in \mathbb{C}$, one declares $f(x, y)=(y, z)$ where $z$ is chosen so that $(0, z)$ lies on the line from $(x, P(x))$ to $(y, P(y))$. This prescription defines a 1-stable meromorphic map $f: \mathbb{P}^{1} \times \mathbb{P}^{1} \rightarrow \mathbb{P}^{1} \times \mathbb{P}^{1}$ with small topological degree.

Proposition 4.4. The secant map $f$ has finite dynamical energy. More precisely, $G^{+} \in \mathcal{E}^{1}\left(T^{-}, \omega^{+}\right)$and $G^{-} \in \mathcal{E}^{1}\left(T^{+}, \omega^{-}\right)$.

Proof. We have that $I^{-}=I^{-}\left(f^{n}\right)=\{(z, z): P(z)=0\}$ consists of fixed points for every $n \in \mathbb{N}$ and that each of these is attracting for $f$. It follows that $\operatorname{dist}\left(f^{n}\left(I^{-}\right), I^{+}\right), \operatorname{dist}\left(f^{-n}\left(I^{+}\right), I^{-}\right) \geq c$ for some $c>0$ and every $n \in \mathbb{N}$. As with the case of $G^{-}$for polynomial maps of $\mathbb{C}^{2}$ then, we infer that $G^{+}$is finite on $I^{-}$ and vice versa. Thus by Corollary 3.5, $G^{+} \in \nabla^{1}\left(T^{-}, \omega^{+}\right)$and $G^{-} \in \nabla^{1}\left(T^{+}, \omega^{-}\right)$.

The classes of $T^{+}$and $T^{-}$are both Kähler, moreover, so from Theorem 3.7 we see that $G^{+} \in \mathcal{E}^{1}\left(T^{-}, \omega^{+}\right)$and $G^{-} \in \mathcal{E}^{1}\left(T^{+}, \omega^{-}\right)$.

4.3. Kodaira dimension zero. When $\operatorname{kod}(X)=0$, we may assume after birational conjugation that $X$ is minimal and $f$ is 1-stable (see [DDG1], Proposition 4.3).

Proposition 4.5. Assume $X$ is a minimal surface with $\operatorname{kod}(X)=0$. Then $f$ satisfies condition $\left(E_{\chi}\right)$ with $\chi(t)=t$. Moreover $\log \operatorname{dist}\left(\cdot, I^{+}\right) \in L^{1}\left(\mu_{f}\right)$.

Proof. We know from [DDG1] that $f$ is non-ramified. Therefore $I^{-}$is empty and (see Proposition 4.10 in [DDG1]) $G^{-}$is continuous on $X$. That is, the hypotheses of Corollary 3.5 are satisfied by both $G^{+}$and $G^{-}$. From Proposition 1.20 , we further have $\log \operatorname{dist}\left(\cdot, I^{+}\right) \in L^{1}\left(\mu_{f}\right)$.

4.4. Irrational rotations. The following examples originate in the work of Favre [F] (see also [B]). Their common feature is the existence of a complex line where $f$ is conjugate to a rotation. Choosing the rotation angle properly allows us to produce functions $G^{ \pm}$that are very singular and therefore useful for testing the sharpness of the energy conditions. 
Example 4.6. Given $a \in \mathbb{C}$, we consider the birational transformation

$$
f:[x: y: t] \in \mathbb{P}^{2} \mapsto\left[y^{2}: a y^{2}+t^{2}-x y: y t\right] \in \mathbb{P}^{2},
$$

For (the all but countably many) parameters $a \in \mathbb{C}$ such that $f$ is 1 -stable on $\mathbb{P}^{2}$, we have $\lambda_{1}(f)=2$ is the degree of the homogeneous polynomials defining $f$. Thus $f$ has small topological degree. Moreover, since $\operatorname{dim} H^{1,1}\left(\mathbb{P}^{2}\right)=1$, it follows that we may take $\omega=\omega^{+}=\omega^{-}$. So there are no spurious points in either $I^{+}$or $I^{-}$.

One checks that $I^{+}=\{[1: 0: 0]\}, I^{-}=\{[0: 1: 0]\}$, and that the line $L:=(t=0)$ joining these two points is $f$-invariant. When $a \in \mathbb{C} \backslash[-2,2]$, we have $\overline{I_{\infty}^{+}} \cap \overline{I_{\infty}^{-}}=\emptyset$, where

$$
I_{\infty}^{+}=\bigcup_{n \geq 0} I^{+}\left(f^{n}\right) \quad \text { and } \quad I_{\infty}^{-}=\bigcup_{n \geq 0} I^{-}\left(f^{n}\right) .
$$

This implies that $f$ is 1 -stable and that $G^{ \pm}$is finite at points in $I^{\mp}$. From Proposition 3.2, Corollary 3.5 and Theorem 3.7 it follows that $f$ satisfies condition $\left(E_{\chi}\right)$ for $\chi(t)=t$.

Now suppose that $a=2 \cos \pi \theta \in[-2,2]$. In this case $f_{L}:=f_{\mid L}$ is conjugate to a rotation of angle $2 \pi \theta$, and $f$ is 1 -stable if and only if $\theta$ is irrational. Determining whether or not $f$ satisfies $\left(E_{\chi}\right)$ for any given $\chi$ is tricky. We will show for any given $p \in(0,1)$ that $G^{ \pm} \in \mathcal{E}^{p}\left(T^{\mp}, \omega^{ \pm}\right)$if $\theta$ is not too well approximated by rational numbers. In $[\mathrm{F}]$ it was precisely proved that $G^{ \pm} \notin \mathcal{E}^{1}\left(T^{\mp}, \omega^{ \pm}\right)$for certain values of $\theta$.

Observe that $f$ is conjugate to $f^{-1}$ by the involution $(x, y) \mapsto(y, x)$. Hence by Corollary 3.6 (also Proposition 3.2 and Theorem 3.7), we need only verify that $\lim \sup _{t \rightarrow-\infty}\left|m^{-}(t) \| t\right|^{q-1} d t<+\infty$, where $p$ is some number larger than $q$ and $m^{-}(t)$ is the mean value of $G^{-}$on the sphere $\partial B_{[1: 0: 0]}\left(e^{t}\right)$. By plurisubharmonicity, $m^{-}(t)$ is comparable to $\sup _{B\left(I^{+}, e^{t}\right)} G^{-}$, which is in turn bounded below by $m_{L}^{-}(t):=$ $\sup _{B\left(I^{+}, e^{t}\right) \cap L} G^{-}$. We therefore estimate the latter, fixing coordinates on $L \cong \mathbb{P}^{1}$ so that $f_{L}(x)=e^{2 \pi i \theta} x$ for all $x \in \mathbb{C} \subset L$ and that $I^{+}, I^{-}$become the points 1 and -1 , respectively. Hence

$$
G^{-}(x) \simeq \sum_{n \geq 0} 2^{-n} \log \left|e^{2 \pi i n \theta} x+1\right| .
$$

Note for any $n \in \mathbb{N}$ and $t>0$ that $\sup _{|x-1|=e^{-t}} \log \left|e^{2 \pi i n \theta} x+1\right|$ is essentially achieved at $x=1+e^{-t}$. Hence

$$
m_{L}^{-}(t) \approx \sum_{n \geq 0} 2^{-n} \max \{\log \epsilon(n), t\}
$$

where $\epsilon(n):=\min _{m \in \mathbb{Z}}|2 n \theta-(2 m+1)|$. Now let $\left(\frac{2 m_{j}+1}{2 n_{j}}\right)_{j \in \mathbb{N}} \subset \mathbb{Q}$ be the sequence uniquely determined by requiring $\operatorname{gcd}\left(2 n_{j}, 2 m_{j}+1\right)=1$, setting $n_{0}=1$ 
and choosing $n_{j}>n_{j-1}$ to be the smallest integer such that $\epsilon\left(n_{j}\right)<\epsilon\left(n_{j-1}\right)$. From this it is entertaining to compute that

$$
\lim \sup \left|m_{L}^{-}(t)\right||t|^{q-1} d t \approx \lim \sup 2^{-n_{j}}\left|\log \epsilon\left(n_{j}\right)\right|^{q} .
$$

In particular, $G^{ \pm} \in \mathcal{E}^{p}\left(T^{\mp}, \omega^{ \pm}\right)$if the right side is finite for some $q>p$. We remark that finiteness holds for almost all $\theta \in \mathbb{R}$ and can be checked for any given irrational $\theta$ by examining its continued fraction expansion.

The next example is studied in [DG] where it is proved that $G^{+} \in L^{1}\left(T^{-}\right)$. Hence we are in a situation where the alternative of Theorem 2.3 holds.

Example 4.7. For parameters $a, b, c \in \mathbb{C}^{*}$, we consider the rational transformation of the complex projective plane, $f=f_{a b c}: \mathbb{P}^{2} \rightarrow \mathbb{P}^{2}$, defined by

$f[x: y: z]=[b c x(-c x+a c y+z): a c y(x-a y+a b z): a b z(b c x+y-b z)]$.

The following facts can be verified by straightforward computation.

- $f_{a b c}$ is birational with inverse $f^{-1}=f_{a^{-1} b^{-1} c^{-1}}$.

- $I_{f}=\{[a: 1: 0],[0: b: 1],[1: 0: c]\}$.

- $f$ preserves each of the lines $\{x=0\},\{y=0\},\{z=0\}$ according to the formulas

$$
\begin{gathered}
{[x: 1: 0] \mapsto[-b c x: a: 0], \quad[0: y: 1] \mapsto[0:-a c y: b],} \\
{[1: 0: z] \mapsto[c: 0:-b a z]}
\end{gathered}
$$

In particular, we have $I_{f}^{\infty}, I_{f-1}^{\infty} \subset\{x y z=0\}$ for all $a, b, c \in \mathbb{C}^{*}$.

Given $s>1$ and an irrational number $\theta \in \mathbb{R}$, let $f: \mathbb{P}^{2} \rightarrow \mathbb{P}^{2}$ be the birational map $f=f_{a b c}$ with $a=i, b=-s e^{2 \pi i \theta}, c=i / s$. One can then check (see [DG]) that

- $f$ is 1-stable on $X=\mathbb{P}^{2}$;

- $T^{+} \in L^{1}\left(T^{-}\right)$.

Thus the measure $\mu_{f}=T^{+} \wedge T^{-}$is a well defined probability measure. It is further shown in [DG] that $\mu_{f}$ does not charge curves and is mixing. We can apply the alternative of Theorem 2.3, reinforced by the ergodicity of $\mu_{f}$ :

- either $\mu_{f}$ is supported on the pluripolar set $\left\{G^{+}+G^{-}=-\infty\right\}$,

- or $f$ has finite dynamical energy.

The latter almost always occurs ${ }^{2}$ : when $\theta$ is not too close to rational numbers, this can be verified by arguing as in the previous example.

\footnotetext{
${ }^{2}$ But not always, see [Bu].
} 


\section{References}

[B] E. Bedford, On the dynamics of birational mappings of the plane. J. Korean Math. Soc. 40 (2003), no. 3, 373-390. Zbl 1111.37303 MR 1973907312

[BD] E. Bedford and J. Diller, Energy and invariant measures for birational surface maps. Duke Math. J. 128 (2005), no. 2, 331-368. Zbl 1076.37031 MR 2140266 280, 294, 304, 306

[BT1] E. Bedford and B. A. Taylor, A new capacity for plurisubharmonic functions. Acta Math. 149 (1982), no. 1-2, 1-40. Zbl 0547.32012 MR 0674165 279, 286, 293

[BT2] E. Bedford and B. A. Taylor, Fine topology, Šilov boundary, and $\left(d d^{c}\right)^{n}$. J. Funct. Anal. 72 (1987), no. 2, 225-251. Zbl 0677.31005 MR 0886812282

[BE] H. Ben Messaoud and H. ElMir, Opérateur de Monge-Ampère et tranchage des courants positifs fermés. J. Geom. Anal. 10 (2000), 139-168. Zbl 1005.32023 MR 1758586301

$[\mathrm{Bu}] \mathrm{X}$. Buff, Courants dynamiques pluripolaires. Ann. Fac. Sci. Toulouse, to appear. arXiv:1003.2219v1 [math.DS] 281, 314

[CGZ] D. Coman, V. Guedj, and A. Zeriahi, Domains of definition of Monge-Ampère operators on compact Kähler manifolds. Math. Z. 259 (2008), no. 2, 393-418. Zbl 1137.32015 MR 2390088284

[De] J.-P. Demailly, Monge-Ampère operators, Lelong numbers and intersection theory. In Complex analysis and geometry, Univ. Ser. Math., Plenum Press, New York 1993, 115-193. Zbl 0792.32006 MR 1211880 287, 301

[DG] J. Diller and V. Guedj, Regularity of dynamical Green functions. Trans. Amer. Math. Soc. 361 (2009), 4783-4805. Zbl 1172.32004 MR 2506427 280, 281, 314

[DDG1] J. Diller, R. Dujardin, and V. Guedj, Dynamics of meromorphic maps with small topological degree I: from cohomology to currents. Indiana Univ. Math. J. 59 (2010), no. 2, 521-561. Zbl 05808404 MR 2648077 277, 278, 279, 296, 297, 299, 300, 303, $304,308,309,310,312$

[DDG3] J. Diller, R. Dujardin, and V. Guedj, Dynamics of meromorphic maps with small topological degree III: geometric currents and ergodic theory. Ann. Sci. Éc. Norm. Supér. (4) 43 (2010), no. 2, 235-278. Zbl 1197.37059 MR 2662665 278, 279, 297, 300,310

[Du1] R. Dujardin, Structure properties of laminar currents. J. Geom. Anal. 15 (2005), 25-47. Zbl 1076.37033 MR 2132264 297, 300

[Du2] R. Dujardin, Laminar currents and birational dynamics. Duke Math. J. 131 (2006), 219-247. Zbl 1099.37037 MR 2219241 281, 300, 302

[F] C. Favre, Points périodiques d'applications birationnelles de $\mathbf{P}^{2}$. Ann. Inst. Fourier (Grenoble) 48 (1998), no. 4, 999-1023. Zbl 0924.58083 MR 1656005 312, 313

[FJ] C. Favre and M. Jonsson, Dynamical compactifications of $\mathbf{C}^{2}$. Ann. of Math. 173 (2011), no. 1, 211-249. 310, 311

[GZ1] V. Guedj and A. Zeriahi, Intrinsic capacities on compact Kähler manifolds. J. Geom. Anal. 15 (2005), no. 4, 607-639. Zbl 1087.32020 MR 2203165284 
[GZ2] V. Guedj and A. Zeriahi, The weighted Monge-Ampère energy of quasiplurisubharmonic functions. J. Funct. Anal. 250 (2007), 442-482. Zbl 1143.32022 MR 2352488 277, 279, 284, 288, 290, 309

[Le] P. Lelong, Fonctions entières ( $n$ variables) et fonctions plurisousharmoniques d'ordre fini dans $\mathbf{C}^{n}$. J. Analyse Math. 12 (1964), 365-407. Zbl 0126.29602 MR 0166391 301

[KaHa] A. Katok and B. Hasselblatt, Introduction to the modern theory of dynamical systems. Cambridge University Press, Cambridge 1995. Zbl 0878.58020 MR 1326374281

Received October 21, 2008

Jeffrey Diller, Department of Mathematics, University of Notre Dame, Notre Dame, IN 46556, U.S.A.

E-mail: diller.1@nd.edu

Romain Dujardin, UFR de Mathématiques et Institut de Mathématiques de Jussieu, Université Paris Diderot, 175 rue du Chevaleret, 75013 Paris, France

E-mail: dujardin@math.jussieu.fr

Vincent Guedj, Université Aix-Marseille 1, LATP, 13453 Marseille Cedex 13, France

E-mail: guedj@cmi.univ-mrs.fr 